\title{
Surviving in a metastable de Sitter space-time
}

\author{
Sitender Pratap Kashyap, ${ }^{a}$ Swapnamay Mondal, ${ }^{a}$ Ashoke Sen ${ }^{a, b}$ \\ and Mritunjay Verma ${ }^{a, c}$ \\ ${ }^{a}$ Harish-Chandra Research Institute, \\ Chhatnag Road, Jhusi, Allahabad 211019, India \\ ${ }^{b}$ School of Physics, Korea Institute for Advanced Study, \\ Seoul 130-722, Korea \\ ${ }^{c}$ International Centre for Theoretical Sciences, \\ Malleshwaram, Bengaluru 560 012, India. \\ E-mail: sitenderpratap@mri.ernet.in, swapno@mri.ernet.in, \\ sen@mri.ernet.in, mritunjayverma@mri.ernet.in
}

ABSTRACT: In a metastable de Sitter space any object has a finite life expectancy beyond which it undergoes vacuum decay. However, by spreading into different parts of the universe which will fall out of causal contact of each other in future, a civilization can increase its collective life expectancy, defined as the average time after which the last settlement disappears due to vacuum decay. We study in detail the collective life expectancy of two comoving objects in de Sitter space as a function of the initial separation, the horizon radius and the vacuum decay rate. We find that even with a modest initial separation, the collective life expectancy can reach a value close to the maximum possible value of 1.5 times that of the individual object if the decay rate is less than $1 \%$ of the expansion rate. Our analysis can be generalized to any number of objects, general trajectories not necessarily at rest in the comoving coordinates and general FRW space-time. As part of our analysis we find that in the current state of the universe dominated by matter and cosmological constant, the vacuum decay rate is increasing as a function of time due to accelerated expansion of the volume of the past light cone. Present decay rate is about 3.7 times larger than the average decay rate in the past and the final decay rate in the cosmological constant dominated epoch will be about 56 times larger than the average decay rate in the past. This considerably weakens the lower bound on the half-life of our universe based on its current age.

KEYWORDS: dS vacua in string theory, Cosmology of Theories beyond the SM

ARXIV EPRINT: 1506.00772 


\section{Contents}

1 Introduction 1

2 Independent decay $\quad 5$

3 Vacuum decay in $\mathbf{1 + 1}$ dimensional de Sitter space $\quad 5$

$\begin{array}{ll}3.1 \text { Isolated comoving object } & 6\end{array}$

$\begin{array}{lll}3.2 & \text { A pair of comoving objects } & 7\end{array}$

4 Vacuum decay in 3+1 dimensional de Sitter space $\quad 14$

$\begin{array}{lll}4.1 & \text { Isolated comoving object } & 14\end{array}$

$\begin{array}{ll}4.2 \text { A pair of comoving objects } & 15\end{array}$

$\begin{array}{ll}4.3 \text { The case of small initial separation } & 19\end{array}$

5 Generalizations $\quad 20$

5.1 Multiple objects in de Sitter space 20

$\begin{array}{lll}5.2 & \text { Realistic trajectories } & 22\end{array}$

$\begin{array}{ll}5.3 \text { Matter effect } & 22\end{array}$

$\begin{array}{llr}6 & \text { Discussion } & 25\end{array}$

A Decay rate for equation of state $p=w \rho \quad 28$

\section{Introduction}

The possibility that we may be living in a metastable vacuum has been explored for more that fifty years [1-6]. Discovery of the accelerated expansion of the universe $[7,8]$ and subsequent developments in string theory leading to the construction of de Sitter vacua [911] suggest that the vacuum we are living in at present is indeed metastable. Unfortunately our understanding of string theory has not reached a stage where we can make a definite prediction about the decay rate of our vacuum. The only information we have about this is from the indirect observation that our universe is about $1.38 \times 10^{10}$ years old. Therefore, assuming that we have not been extremely lucky we can conclude that our inverse decay rate $^{1}$ is at least of the same order. ${ }^{2}$

\footnotetext{
${ }^{1}$ For exponential decay the inverse decay rate differs from half-life by a factor of $\ln 2$. In order to simplify terminology, we shall from now on use only inverse decay rate and life expectancy — to be defined later as measures of longevity.

${ }^{2}$ We shall in fact see in section 5.3 that the actual lower bound for the current inverse decay rate is weaker by a factor of 3.7, making it comparable to the time over which the earth will be destroyed due to the increase in the size of the sun. Allowing for the possibility that we could have been extremely lucky reduces the lower bound on the inverse decay rate by about a factor of $10[12,13]$.
} 
Typically the decay of a metastable vacuum proceeds via bubble nucleation [1-6] (see [14] for a recent survey). In a small region of space-time the universe makes transition to a more stable vacuum, and this bubble of stable vacuum ${ }^{3}$ then expands at a speed that asymptotically approaches the speed of light, converting the rest of the region it encounters also to this stable phase. Due to this rapid expansion rate it is impossible to observe the expanding bubble before encountering it - it reaches us when we see it. However, due to the existence of the future horizon in the de Sitter space, even a bubble expanding at the speed of light cannot fill the whole space at future infinity. Indeed, it has been known for quite some time that in de Sitter space if the expansion rate of the universe exceeds the decay rate due to phase transition then even collectively the bubbles of stable vacuum cannot fill the whole space [15] and there will always be regions which will continue to exist in the metastable vacuum. Nevertheless, any single observer in the metastable vacuum will sooner or later encounter an expanding bubble of stable vacuum, and the probability of this decay per unit time determines the inverse decay rate of the observer in the metastable vacuum.

This suggests that while any single observer will always have a limited average life span determined by the microscopic physics, a civilization could collectively increase its longevity by spreading out and establishing different civilizations in different parts of the universe [16]. If the bubble of stable vacuum hits the civilization - henceforth refered to as object - in the initial stages of spreading out then it does not help since the same bubble will most likely destroy all the objects. However, with time the different objects will go outside each other's horizon and a single bubble of stable vacuum will not be able to destroy all of them. This will clearly increase the life expectancy of the objects collectively - defined as the average value of the time at which the last surviving object undergoes vacuum decay - although there will be no way of telling a priori which one will survive the longest. A simple calculation shows that if we could begin with 2 objects already far outside each other's horizon so that their decay probabilities can be taken to be independent, then the life expectancy of the combined system increases by a factor of $3 / 2$ compared to the life expectancy of a single isolated object. In the case of $n$ copies the life expectancy increases by a factor given by the $n$-th harmonic number. However, in actual practice we cannot begin with copies of the object already outside each other's horizon. As a result the increase in the life expectancy is expected to be lower.

The goal of this paper will be to develop a systematic procedure for computing the increase in the life expectancy of the object as a result of making multiple copies of itself. For two objects we obtain explicit expression for the life expectancy in terms of three parameters: the Hubble constant $H$ of the de Sitter space-time determined by the cosmological constant, the vacuum decay rate or equivalently the life expectancy $T$ of a single isolated object and the initial separation $r$ between the two objects. In fact due to dimensional

\footnotetext{
${ }^{3}$ We shall refer to the more stable vacuum as the stable vacuum, even if this vacuum in turn could decay to other vacua of lower energy density. In any case since this vacuum will have negative cosmological constant, the space-time inside the bubble will undergo a gravitational crunch [6]. We shall ignore the possibility of decay to Minkowski vacua or other de Sitter vacua of lower cosmological constant since the associated decay rates are very small due to smallness of the cosmological constant of our vacuum.
} 


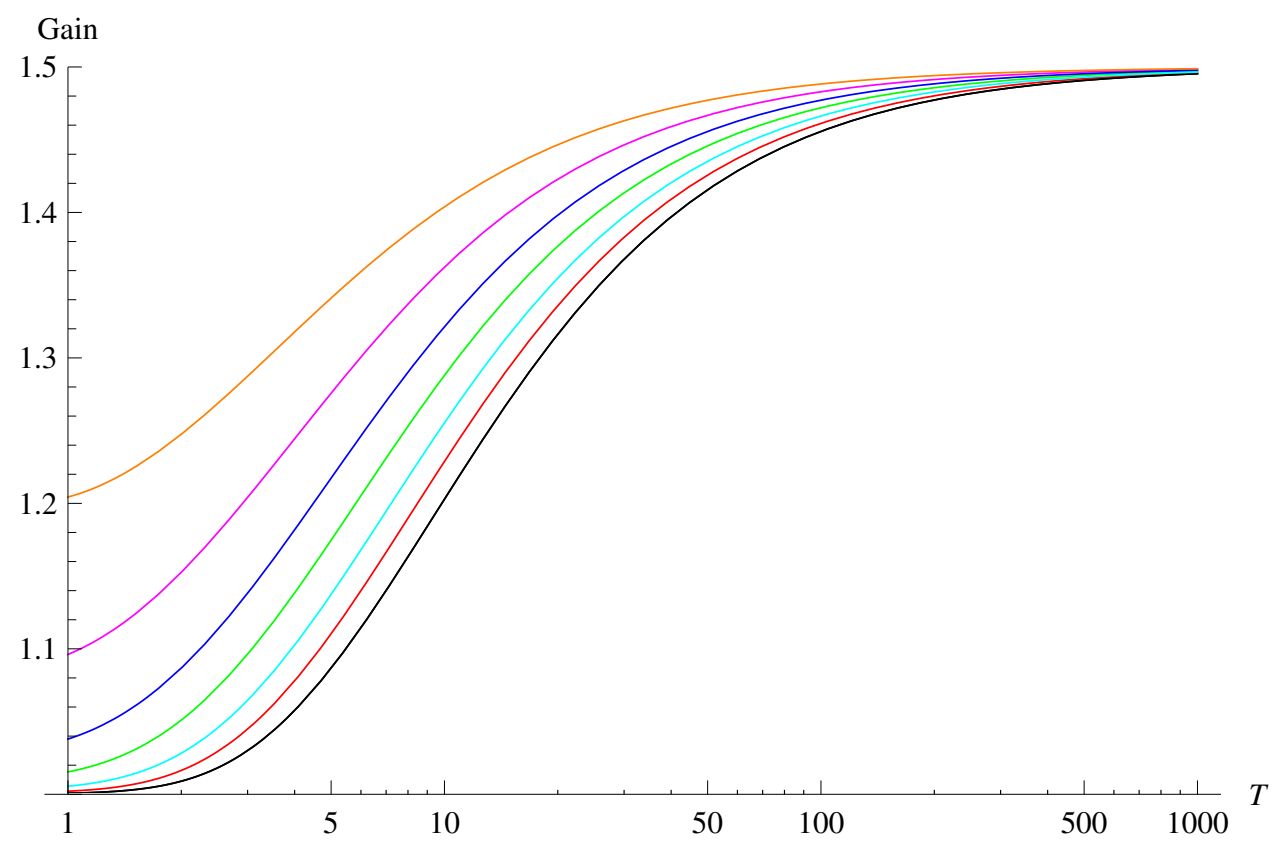

Figure 1. The figure showing the 'gain' in the life expectancy for two objects compared to that of one object as a function of $T$ for $r=.0003, .001, .003, .01, .03, .1$ and .3 .

reasons the result depends only on the combination $H T$ and $H r$, so we work by setting $H=1$. In figure 1 we have shown the result for the ratio of the life expectancy of two objects to that of a single object — called the 'gain' - as a function of $T$ for different choices of $r$. From this we see that even for a modest value of $r=3 \times 10^{-4}$ the gain in the life expectancy reaches close to the maximum possible value of 1.5 if $T$ is larger that 100 times the horizon size of the de Sitter space, i.e. the decay rate is less than $1 \%$ of the expansion rate. $T=100$ corresponds to about $1.7 \times 10^{12}$ years. $r=3 \times 10^{-4}$ corresponds to a physical distance of the order of $5 \times 10^{6}$ light years and is of the order of the minimal distance needed to escape the local gravitationally bound system of galaxies. If $T=10-$ i.e. of order $1.7 \times 10^{11}$ years - the gain is about $20 \%$ for $r=3 \times 10^{-4}$. These time scales are shorter than the time scale by which all the stars in the galaxy will die. Therefore, if $T$ lies between $10^{11}$ years and the life span of the last star in the local group of galaxies which will be gravitationally bound and will remain inside each other's horizon, then we gain a factor of $1.2-1.5$ in life expectancy even by making one additional copy of the object at a distance larger than about $10^{7}$ light years from us. On the other hand if $T$ is larger than the life span of the last star in the galaxy then our priority should be to plan how to survive the death of the galaxy rather than vacuum decay. Some discussion on this can be found in [17].

Even though most of our analysis focusses on the case of a pair of objects in de Sitter space at fixed comoving coordinates, our method is quite general and can be applied to arbitrary number of objects in a general FRW metric moving along general trajectories. We discuss these generalizations in section 5. In particular considering the case of a single object in an FRW metric dominated by matter and cosmological constant, as is the case 


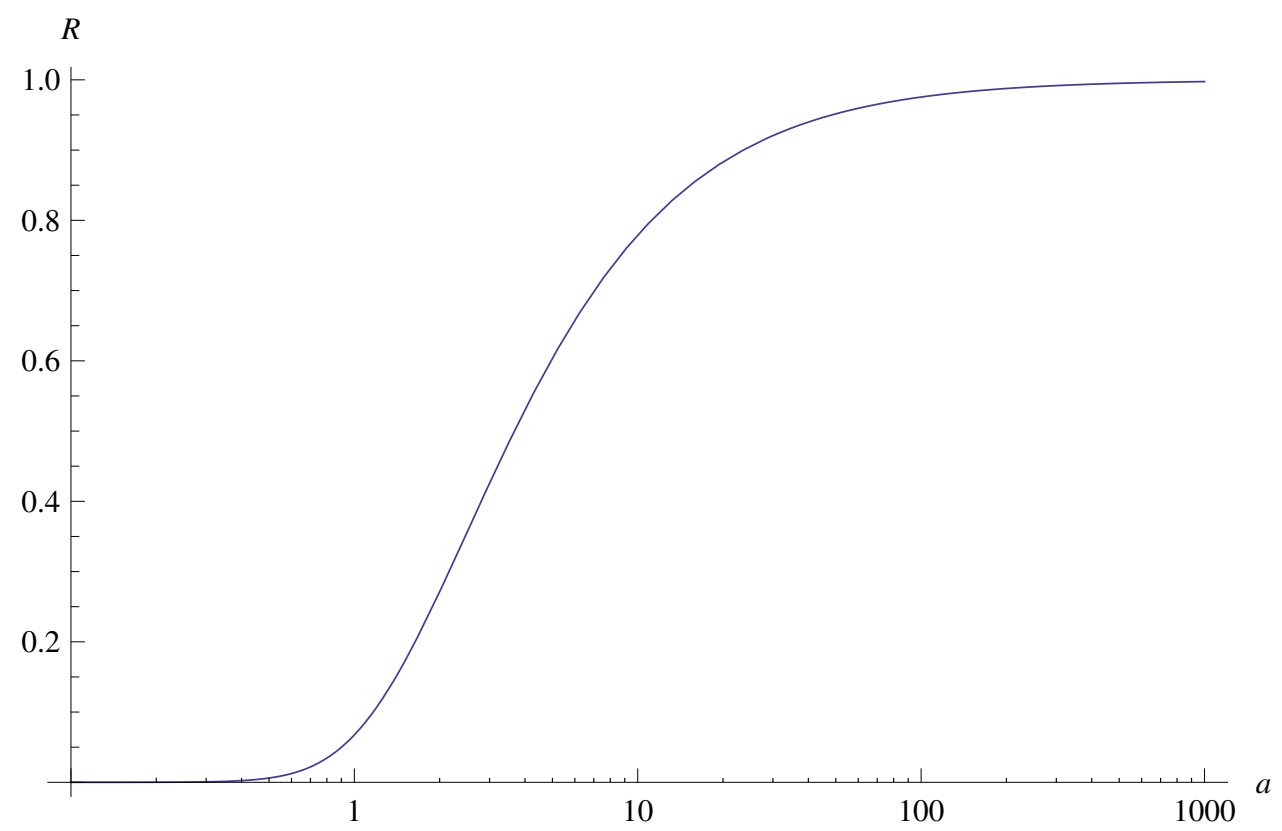

Figure 2. Growth of the relative decay rate $R$ - defined as the ratio of the decay rate to its asymptotic value - of a single object in FRW space-time as a function of the scale factor $a$. Value of $R$ at $a=1$ represents the decay rate today relative to what it would be in the cosmological constant dominated epoch.

with the current state of our universe, we find that the vacuum decay rate increases as a function of time due to accelerated expansion of the volume of the past light cone. This has been shown in figure 2. This rate approaches a constant value as the universe enters the cosmological constant dominated era, but we find for example that this asymptotic decay rate is about 15 times larger than the decay rate today, which in turn is about 3.7 times larger than the average decay rate in the past. Now given that the universe has survived for about $1.38 \times 10^{10}$ years, we can put a lower bound of this order on the inverse of the average decay rate in the past. ${ }^{4}$ This translates to a lower bound of order $3.7 \times 10^{9}$ years on the inverse decay rate today and $2.5 \times 10^{8}$ years on the asymptotic inverse decay rate.

The rest of the paper is organised as follows. In section 2 we describe the case of the decay of $n$ objects assuming that their decay probabilities are independent of each other, and show that the life expectancy of the combined system goes up by a factor equal to the $n$-th harmonic number. In section 3 we carry out the complete analysis for two observers in $1+1$ dimensional de Sitter space. The final result for the life expectancy of the combined system can be found in (3.30). This is generalized to the case of two observers in $3+1$ dimensional de Sitter space-time in section 4. Eq. (4.17) together with (4.16) and (4.11) gives the probability that at least one of the two objects survives till time $t$, which can then be used to compute the life expectancy of the combined system using (4.18). In section 5 we discuss various generalizations including the case of multiple observers, general trajectories

\footnotetext{
${ }^{4}$ One must keep in mind that this is not a strict bound since we could have survived till today by just being lucky.
} 
and general FRW type metric. We conclude in section 6 with a discussion of how in future we could improve our knowledge of possible values of the parameters $r$ and $T$ which enter our calculation. In appendix A we compute the time dependence of the decay rate for a general equation of state of the form $p=w \rho$.

\section{Independent decay}

Let us suppose that we have two independent objects, each with a decay rate of $c$ per unit time. We shall label them as $C_{1}$ and $C_{2}$. If we begin with the assumption that both objects exist at time $t=0$ then the probability that the first object exists after time $t$ is

$$
P_{1}(t)=e^{-c t} .
$$

Therefore, the probability that it decays between time $t$ and $t+\delta t$ is $-\dot{P}_{1}(t) \delta t$ where $\dot{P}_{1}$ denotes the derivative of $P_{1}$ with respect to $t$, and its life expectancy, is

$$
\bar{t}_{1}=-\int_{0}^{\infty} t \dot{P}_{1}(t) d t=\int_{0}^{\infty} P_{1}(t) d t=c^{-1} .
$$

Independently of this the probability that the second object exists after time $t$ is also given by $\exp [-c t]$ and it has the same life expectancy.

Now let us compute the life expectancy of both objects combined, defined as the average of the larger of the actual life time of $C_{1}$ and $C_{2}$. To compute this note that since the two objects are independent, the probability that both will decay by time $t$ is given by $\left(1-P_{1}(t)\right)\left(1-P_{2}(t)\right)=\left(1-P_{1}(t)\right)^{2}$. Therefore, the probability that the last one to survive decays between $t$ and $t+\delta t$ is $\frac{d}{d t}\left(1-P_{1}(t)\right)^{2} \delta t$. This gives the life expectancy of the combined system to be

$$
\bar{t}_{12}=\int_{0}^{\infty} t \frac{d}{d t}\left(1-P_{1}(t)\right)^{2} d t=\frac{3}{2} c^{-1}
$$

Therefore, we see that by taking two independent objects we can increase the life expectancy by a factor of $3 / 2$. A similar argument shows that for $n$ independent objects the life expectancy will be

$$
\bar{t}_{12 \cdots n}=\int_{0}^{\infty} t \frac{d}{d t}\left(1-P_{1}(t)\right)^{n} d t=\left(1+\frac{1}{2}+\frac{1}{3}+\cdots+\frac{1}{n}\right) c^{-1} .
$$

\section{Vacuum decay in $1+1$ dimensional de Sitter space}

Consider $1+1$ dimensional de Sitter space

$$
d s^{2}=-d t^{2}+e^{2 t} d x^{2} .
$$

Note that we have set the Hubble constant of the de Sitter space and the speed of light to unity so that all other time / lengths appearing in the analysis are to be interpreted as 


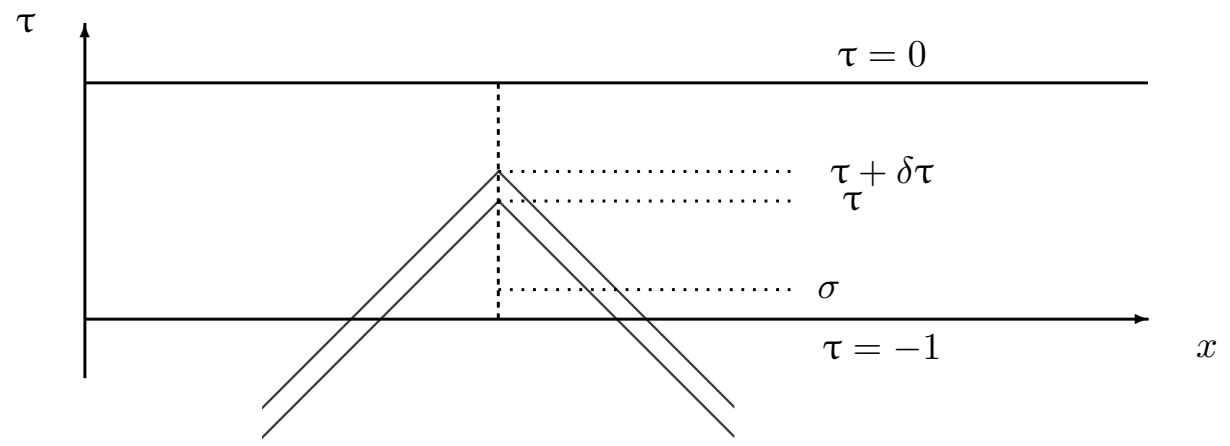

Figure 3. A comoving object in de Sitter space and its past light cones at conformal times $\tau$ and $\tau+\delta \tau$.

their values in units of the inverse Hubble constant. We introduce the conformal time $\tau$ via

$$
\tau=-e^{-t}
$$

in terms of which the metric takes the form

$$
d s^{2}=\tau^{-2}\left(-d \tau^{2}+d x^{2}\right) .
$$

At $t=0$ we have $\tau=-1$ and comoving distances coincide with the physical distances.

We shall use this space-time as a toy model for studying the kinematics of vacuum decay. We shall assume that in this space-time there is a certain probability per unit time per unit volume of producing a bubble of stable vacuum, which then expands at the speed of light causing decay of the metastable vacuum. We shall not explore how such a bubble is produced; instead our goal will be to study its effect on the life expectancy of the objects living in this space. In section 4 we shall generalize this analysis to $3+1$ dimensional de Sitter space.

\subsection{Isolated comoving object}

Consider a single object in de Sitter space at rest in the comoving coordinate $x$ (say at $x=0)$, shown by the vertical dashed line in figure 3 . We start at $t=0(\tau=-1)$ and are interested in calculating the probability that it survives at least till conformal time $\tau$. If we denote this by $P_{0}(\tau)$ then the probability that it will decay between $\tau$ and $\tau+\delta \tau$ is $-P_{0}^{\prime}(\tau) \delta \tau$ where ${ }^{\prime}$ denotes derivative with respect to $\tau$. On the other hand this probability is also given by the product of $P_{0}(\tau)$ and the probability that a vacuum bubble is produced somewhere in the past light cone of the object between $\tau$ and $\tau+\delta \tau$, as shown in figure 3 . The volume of the past light cone of this interval can be easily calculated to be

$$
2 \delta \tau \int_{-\infty}^{\tau} \frac{d \sigma}{\sigma^{2}}=-\frac{2}{\tau} \delta \tau
$$

Therefore, if $K$ is the probability of producing the bubble per unit space-time volume then the probability of producing a bubble in the past light cone of the object between $\tau$ and 


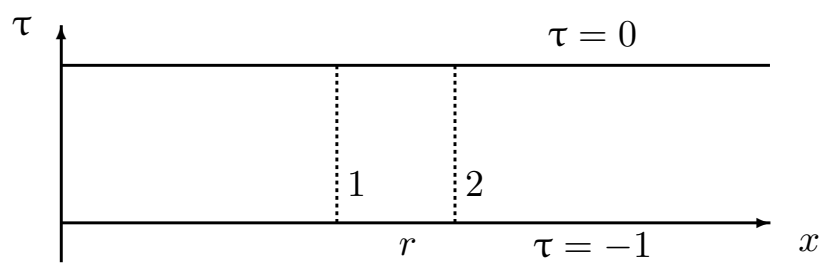

Figure 4. Two comoving objects in de Sitter space separated by physical distance $r$ at $\tau=-1$.

$\tau+\delta \tau$ is given by $-2 K \delta \tau / \tau$. The previous argument then leads to the equation

$$
P_{0}^{\prime}(\tau)=2 K \tau^{-1} P_{0}(\tau)
$$

This equation, together with the boundary condition $P_{0}(\tau=-1)=1$, can be integrated to give

$$
\ln P_{0}(\tau)=2 K \ln (-\tau) .
$$

In terms of physical time $t$ we have ${ }^{5}$

$$
P_{0}(t)=e^{-2 K t}
$$

From this we can calculate the life expectancy, defined as the integral of $t$ weighted by the probability that the object undergoes vacuum decay between $t$ and $t+d t$. Since the latter is given by $-\dot{P}_{0}(t) d t$, we have the life expectancy

$$
T=-\int_{0}^{\infty} t \dot{P}_{0}(t) d t=\int_{0}^{\infty} P_{0}(t) d t=\frac{1}{2 K},
$$

where in the second step we have used integration by parts. We shall express our final results in terms of $T$ instead of $K$.

\subsection{A pair of comoving objects}

Next we shall consider two comoving objects $C_{1}$ and $C_{2}$ in de Sitter space separated by physical distance $r$ at $t=0$ or equivalently $\tau=-1$. We shall take $r<1$, i.e. assume that the two objects are within each other's horizon at the time they are created. We denote by $P_{i}(\tau)$ the probability that $C_{i}$ survives at least till conformal time $\tau$ for $i=1,2$ and by $P_{12}\left(\tau_{1}, \tau_{2}\right)$ the joint probability that $C_{1}$ survives at least till conformal time $\tau_{1}$ and $C_{2}$ survives at least till conformal time $\tau_{2}$. The boundary condition will be set by assuming that both objects exist at $\tau=-1$, so that we have

$$
P_{1}(-1)=1, \quad P_{2}(-1)=1, \quad P_{12}\left(-1, \tau_{2}\right)=P_{2}\left(\tau_{2}\right), \quad P_{12}\left(\tau_{1},-1\right)=P_{1}\left(\tau_{1}\right) .
$$

First we shall calculate $P_{1}(\tau)$ and $P_{2}(\tau)$. They must be identical by symmetry, so let us focus on $P_{1}(\tau)$. The calculation is similar to that for $P_{0}(\tau)$ above for a single isolated

\footnotetext{
${ }^{5}$ Note that by an abuse of notation we have used the same symbol $P_{0}$ to denote the probability as a function of $t$ although the functional form changes. We shall continue to follow this convention, distinguishing the function by its argument $(t$ or $\tau$ ). Derivatives with respect to $\tau$ and $t$ will be distinguished by using $P_{0}^{\prime}$ to denote $\tau$-derivative of $P_{0}$ and $\dot{P}_{0}$ to denote $t$-derivative of $P_{0}$.
} 


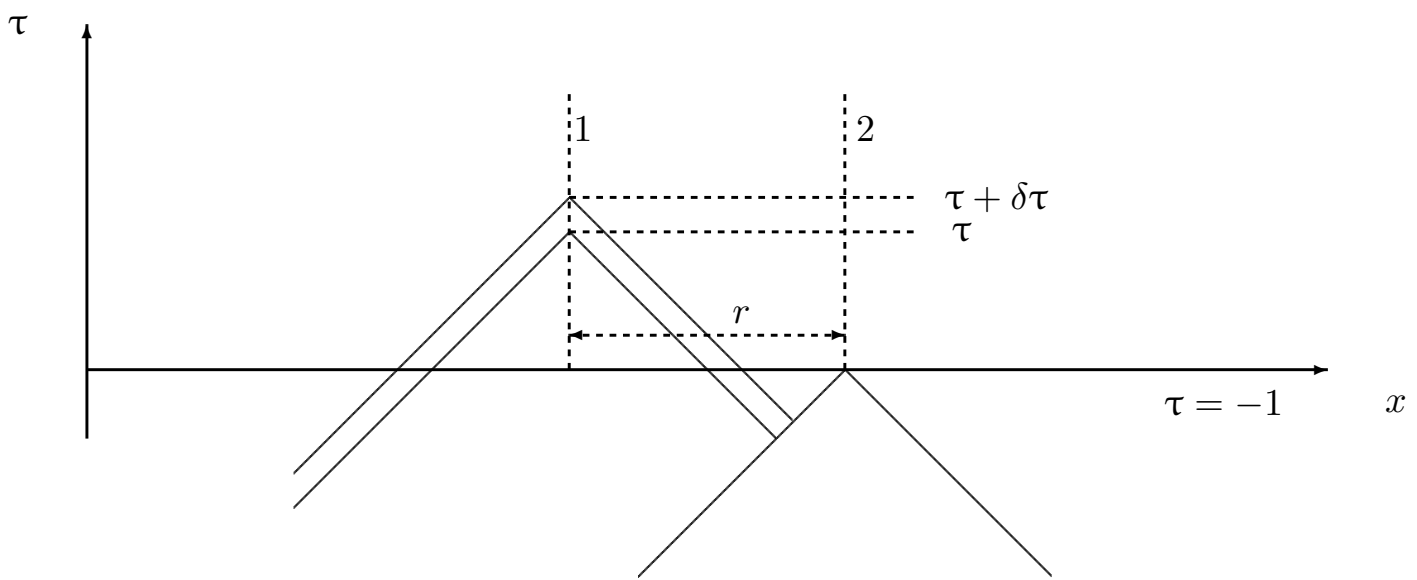

Figure 5. The past light-come of $C_{2}$ at $\tau=-1$ and the past light cone of $C_{1}$ between $\tau$ and $\tau+\delta \tau$ for $\tau<r-1$.

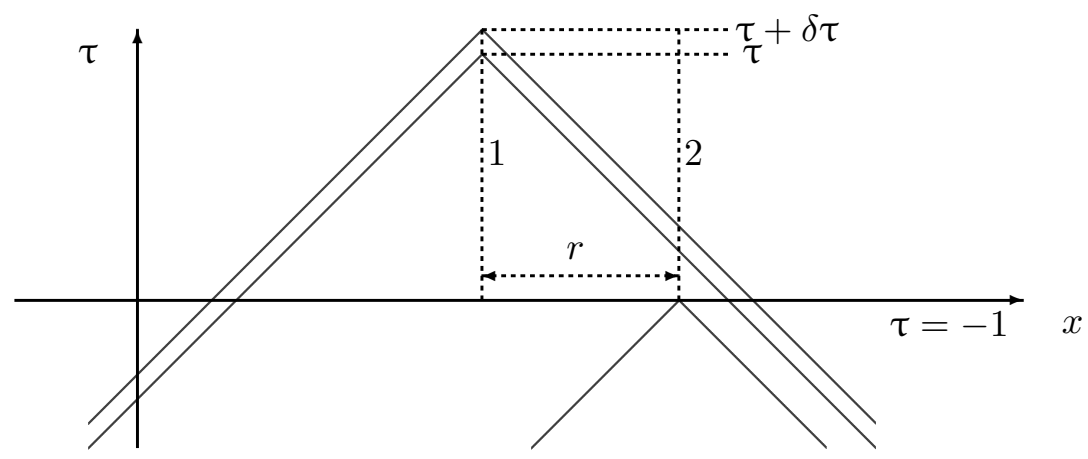

Figure 6. The past light-come of $C_{2}$ at $\tau=-1$ and the past light cone of $C_{1}$ between $\tau$ and $\tau+\delta \tau$ for $\tau>r-1$.

object, except that the existence of $C_{2}$ at $\tau=-1$ guarantees that no vacuum decay bubble wass produced in the past light-come of $C_{2}$ at $\tau=-1$, and hence while computing the volume of the past light cone of the $C_{1}$ between $\tau$ and $\tau+\delta \tau$, we have to exclude the region inside the past light cone of $C_{2}$ at $\tau=-1$. This has been shown in figure 5 . This volume is given by

$$
\delta \tau\left[2 \int_{-\infty}^{\tau} \frac{d \sigma}{\sigma^{2}}-\int_{-\infty}^{-1-\frac{r-1-\tau}{2}} \frac{d \sigma}{\sigma^{2}}\right]=\delta \tau\left[-\frac{2}{\tau}-\frac{2}{r+1-\tau}\right] \quad \text { for } \tau<r-1 .
$$

However, for $\tau>r-1$ the past light cone of $C_{1}$ between $\tau$ and $\tau+\delta \tau$ does not intersect the past light cone of $C_{2}$ at $\tau=-1$ (see figure 6 ), and we get the volume to be

$$
2 \delta \tau \int_{-\infty}^{\tau} \frac{d \sigma}{\sigma^{2}}=-2 \delta \tau \frac{1}{\tau} \quad \text { for } \tau>r-1
$$

This leads to the following differential equation for $P_{1}(\tau)$ :

$$
\frac{1}{P_{1}(\tau)} \frac{d P_{1}}{d \tau}= \begin{cases}-K[-2 / \tau-2 /(r+1-\tau)] & \text { for } \tau<r-1 \\ 2 K / \tau & \text { for } \tau>r-1\end{cases}
$$


Using the boundary condition $P_{1}(-1)=1$ and the continuity of $P_{1}(\tau)$ across $\tau=r-1$ we get

$$
\ln P_{1}(\tau)= \begin{cases}2 K\{\ln (-\tau)-\ln (r+1-\tau)+\ln (r+2)\} & \text { for } \tau<r-1, \\ 2 K\{\ln (-\tau)-\ln 2+\ln (r+2)\} & \text { for } \tau>r-1 .\end{cases}
$$

Using the symmetry between 1 and 2 we also get the same expression for $P_{2}(\tau)$. In terms of the physical time $t$ we have

$$
P_{1}(t)=P_{2}(t)= \begin{cases}e^{-2 K t}\left(r+1+e^{-t}\right)^{-2 K}(r+2)^{2 K} & \text { for } t<-\ln (1-r) \\ ((r+2) / 2)^{2 K} e^{-2 K t} & \text { for } t>-\ln (1-r)\end{cases}
$$

Therefore, the life expectancy of $C_{1}$ is

$$
\begin{aligned}
\bar{t}_{1} & =-\int_{0}^{\infty} t \dot{P}_{1}(t) d t=\int_{0}^{\infty} P_{1}(t) d t \\
& =(r+2)^{2 K}\left[B\left(\frac{1}{2+r} ; 2 K, 0\right)-B\left(\frac{1-r}{2} ; 2 K, 0\right)+\frac{(1-r)^{2 K}}{2^{2 K+1} K}\right]
\end{aligned}
$$

where $B(x ; p, q)$ is the incomplete beta function, defined as

$$
B(x ; p, q)=\int_{0}^{x} t^{p-1}(1-t)^{q-1} d t=\int_{0}^{x /(1-x)} \frac{y^{p-1}}{(1+y)^{p+q}} d y,
$$

the two expressions being related by the transformation $t=y /(y+1)$. In terms of the life expectancy $T=1 / 2 K$ of a single isolated object, we have

$$
\bar{t}_{1}=(r+2)^{1 / T}\left[B\left(\frac{1}{2+r} ; \frac{1}{T}, 0\right)-B\left(\frac{1-r}{2} ; \frac{1}{T}, 0\right)+T \frac{(1-r)^{1 / T}}{2^{1 / T}}\right] .
$$

$C_{2}$ also has the same life expectancy. (3.17) is somewhat larger than $T$, but that is simply a result of our initial assumption that both objects exist at $t=0$. If both objects had started at the same space-time point and then got separated following some specific trajectories, then there would have been a certain probability that one or both of them will decay during the process of separation; this possibility has been ignored here leading to the apparent increase in the life expectancy. However, for realistic values of $r$ and $T$, which corresponds to $r \ll 1$ and $T \gtrsim 1$, the ratio $\bar{t}_{1} / T$ remains close to unity.

Let us now turn to the computation of the joint survival probability $P_{12}\left(\tau_{1}, \tau_{2}\right)$. In this case the probability that the first object undergoes vacuum decay between $\tau_{1}$ and $\tau_{1}+\delta \tau_{1}$ and the second object survives at least till $\tau_{2}$ is given by $-\delta \tau_{1}\left(\partial P_{12}\left(\tau_{1}, \tau_{2}\right) / \partial \tau_{1}\right)$. On the other hand the same probability is given by $K \times P_{12}\left(\tau_{1}, \tau_{2}\right)$ times the volume of the past light-come of $C_{1}$ between $\tau_{1}$ and $\tau_{1}+\delta \tau_{1}$, excluding the region inside the past light cone of $C_{2}$ at $\tau_{2}$. The relevant geometry has been shown in figures 7,8 and 9 for different ranges of $\tau_{1}$ and $\tau_{2}$. The results are as follows: 


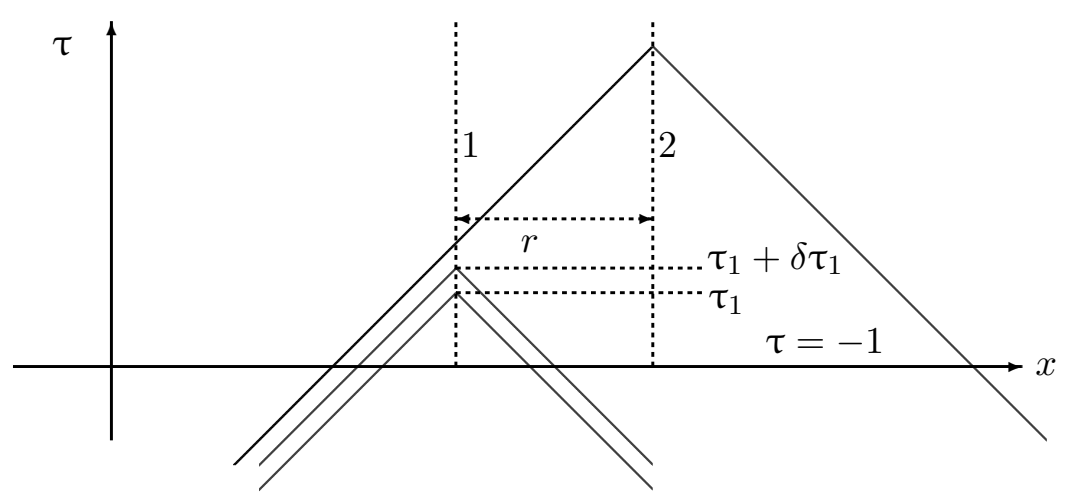

Figure 7. The past light-come of $C_{2}$ at $\tau_{2}$ and the past light cone of $C_{1}$ between $\tau_{1}$ and $\tau_{1}+\delta \tau_{1}$ for $\tau_{1}<\tau_{2}-r$.

1. For $\tau_{1}<\tau_{2}-r$ the geometry is shown in figure 7 . In this case $C_{1}$ at $\tau_{1}$ (and hence the whole of the past light cone of $C_{1}$ between $\tau_{1}$ and $\left.\tau_{1}+\delta \tau_{1}\right)$ is inside the past light cone of $C_{2}$ at $\tau_{2}$. Therefore, the decay probability is zero and we have the equation:

$$
\frac{\partial \ln P_{12}\left(\tau_{1}, \tau_{2}\right)}{\partial \tau_{1}}=0 \quad \text { for } \tau_{1}<\tau_{2}-r
$$

2. For $\tau_{2}-r<\tau_{1}<\tau_{2}+r$ the geometry is as shown in figure 8. In this case $C_{1}$ at $\tau_{1}$ and $C_{2}$ at $\tau_{2}$ are space-like separated. The volume of the past light cone of $C_{1}$ between $\tau_{1}$ and $\tau_{1}+\delta \tau_{1}$ outside the past light cone of $C_{2}$ at $\tau_{2}$ is given by

$$
\int_{-\infty}^{\tau_{1}} \frac{d \sigma}{\sigma^{2}}+\int_{\frac{1}{2}\left(\tau_{1}+\tau_{2}-r\right)}^{\tau_{1}} \frac{d \sigma}{\sigma^{2}}=-\frac{2}{\tau_{1}}-\frac{2}{r-\tau_{1}-\tau_{2}} .
$$

This gives

$$
\frac{\partial \ln P_{12}\left(\tau_{1}, \tau_{2}\right)}{\partial \tau_{1}}=2 K\left\{\frac{1}{\tau_{1}}+\frac{1}{r-\tau_{1}-\tau_{2}}\right\} \quad \text { for } \tau_{2}-r<\tau_{1}<\tau_{2}+r
$$

3. For $0<\tau_{2}+r<\tau_{1}$, the geometry is shown in figure 9 . In this case $C_{2}$ at $\tau_{2}$ is inside the past light cone of $C_{1}$ at $\tau_{1}$ and there is no intersection between the past light cone of $C_{1}$ between $\tau_{1}$ and $\tau_{1}+\delta \tau_{1}$ and the past light cone of $C_{2}$ at $\tau_{2}$. Therefore, the volume of the past light cone of $C_{1}$ between $\tau_{1}$ and $\tau_{1}+\delta \tau_{1}$ is given by

$$
2 \int_{-\infty}^{\tau_{1}} \frac{d \sigma}{\sigma^{2}}=-\frac{2}{\tau_{1}}
$$

and we have

$$
\frac{\partial \ln P_{12}\left(\tau_{1}, \tau_{2}\right)}{\partial \tau_{1}}=2 K \frac{1}{\tau_{1}} \quad \text { for } \tau_{2}+r<\tau_{1}<0 .
$$




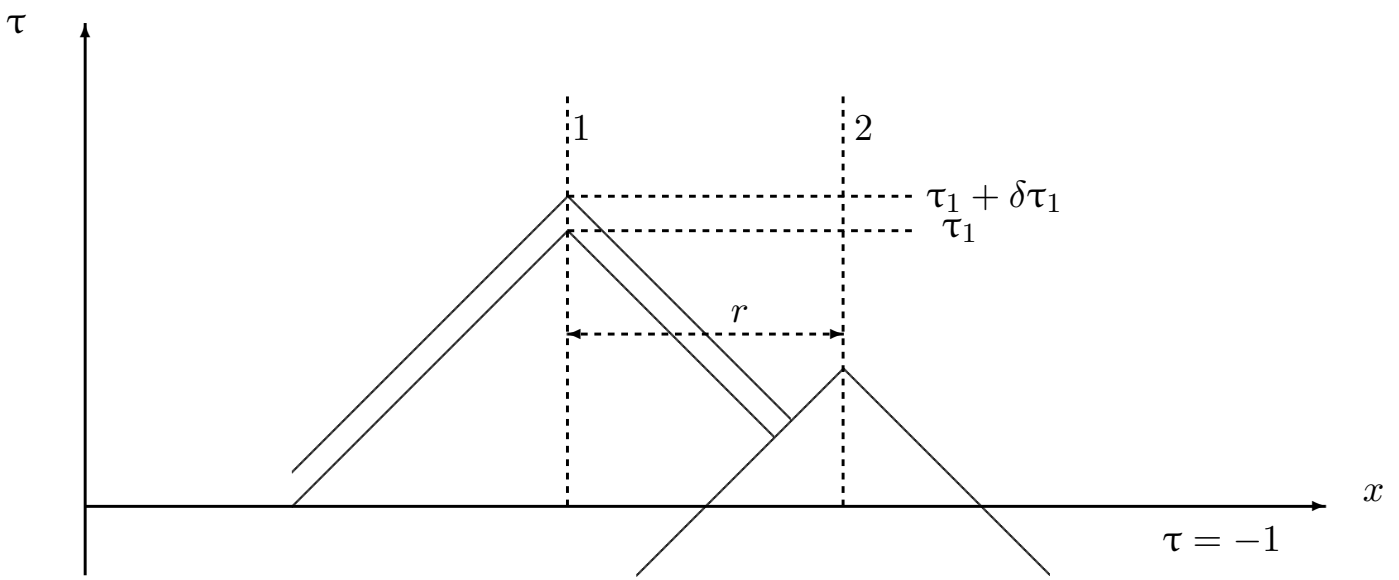

Figure 8. The past light-come of $C_{2}$ at $\tau_{2}$ and the past light cone of $C_{1}$ between $\tau_{1}$ and $\tau_{1}+\delta \tau_{1}$ for $\tau_{2}-r<\tau_{1}<\tau_{2}+r$.

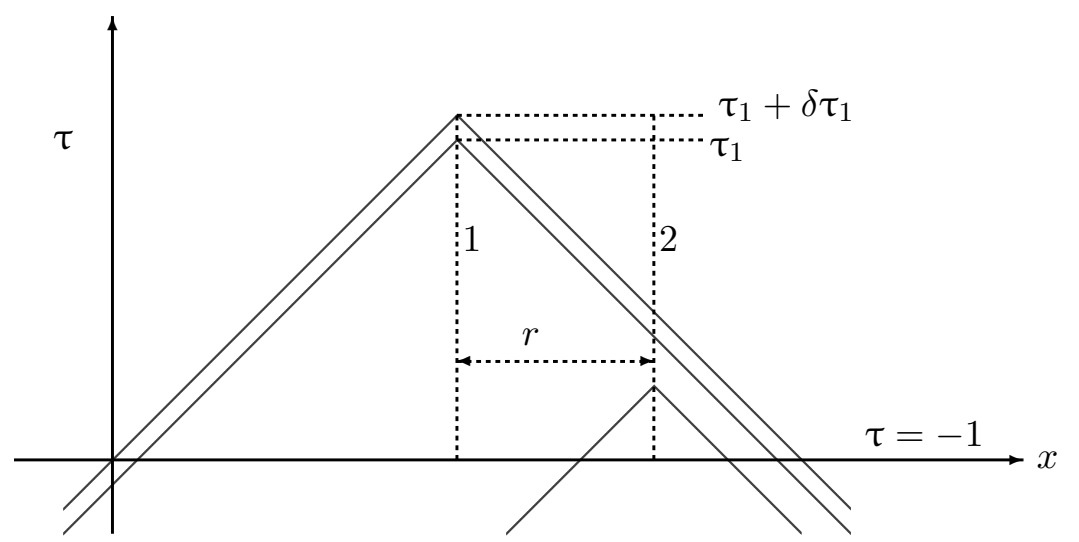

Figure 9. The past light cone of $C_{2}$ at $\tau_{2}$ and the past light cone of $C_{1}$ between $\tau_{1}$ and $\tau_{1}+\delta \tau_{1}$ for $\tau_{2}+r<\tau_{1}<0$.

We can now determine $P_{12}\left(\tau_{1}, \tau_{2}\right)$ by integrating (3.18), (3.20), (3.22) subject to the boundary condition given in (3.9)

$$
P_{12}\left(-1, \tau_{2}\right)=P_{2}\left(\tau_{2}\right)=P_{1}\left(\tau_{2}\right)
$$

and using the fact that $P_{12}\left(\tau_{1}, \tau_{2}\right)$ must be continuous across the subspaces defined by $\tau_{1}=\tau_{2} \pm r$. The result of the integration is

$$
\ln P_{12}\left(\tau_{1}, \tau_{2}\right)=\left\{\begin{array}{r}
2 K\left\{\ln \left(-\tau_{2}\right)+\ln (r+2)-\ln 2\right\} \\
\text { for } \tau_{1}<\tau_{2}-r, \\
2 K\left\{\ln \left(-\tau_{2}\right)+\ln \left(-\tau_{1}\right)-\ln \left(r-\tau_{1}-\tau_{2}\right)+\ln (r+2)\right\}, \\
\text { for } \tau_{2}-r<\tau_{1}<\tau_{2}+r \\
2 K\left\{\ln \left(-\tau_{1}\right)+\ln (r+2)-\ln 2\right\} \\
\text { for } \tau_{2}+r<\tau_{1}<0 .
\end{array}\right.
$$




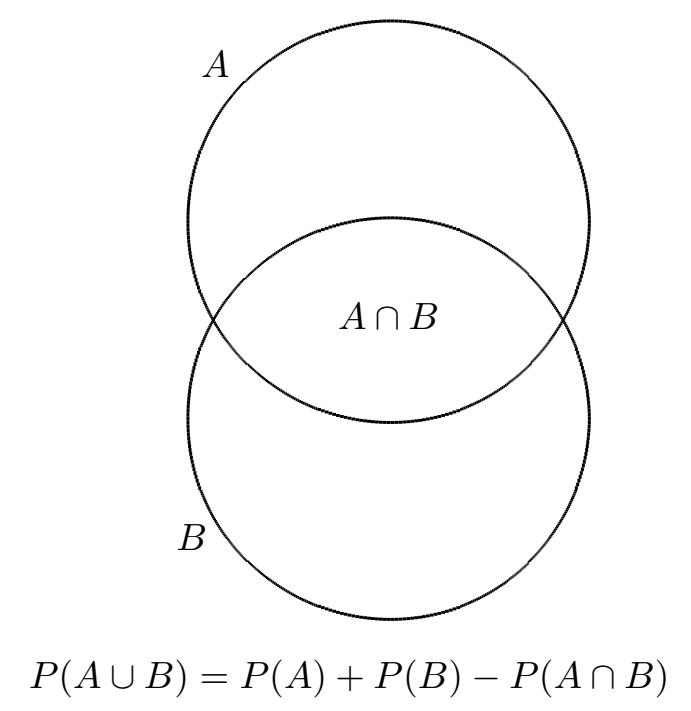

Figure 10. Probability rule for $\mathrm{N}=2$ using Venn Diagram.

Note that the result is symmetric under the exchange of $\tau_{1}$ and $\tau_{2}$ even though at the intermediate stages of the analysis this symmetry was not manifest.

Expressed in terms of physical time the above solution takes the form:

$$
P_{12}\left(t_{1}, t_{2}\right)=\left\{\begin{array}{c}
\{(r+2) / 2\}^{2 K} e^{-2 K t_{2}} \\
\text { for } t_{1}<-\ln \left(r+e^{-t_{2}}\right), \\
(r+2)^{2 K} e^{-2 K\left(t_{1}+t_{2}\right)}\left(r+e^{-t_{1}}+e^{-t_{2}}\right)^{-2 K} \\
\text { for }-\ln \left(r+e^{-t_{2}}\right)<t_{1}<-\ln \left(e^{-t_{2}}-r\right), \\
\{(r+2) / 2\}^{2 K} e^{-2 K t_{1}} \\
\text { for } t_{1}>-\ln \left(e^{-t_{2}}-r\right) .
\end{array}\right.
$$

If $e^{-t_{2}}-r$ is negative then the third case is not relevant and in the second case there will be no upper bound on $t_{1}$. Physically this can be understood by noting that in this case $\tau_{2}>-r$ and $C_{2}$ will never come inside the past light cone of $C_{1}$ even when $\tau_{1}$ reaches its maximum value 0 .

Our interest lies in computing the probability that at least one of the two objects survives till time $t$. Let us denote this by $\widetilde{P}_{12}(t)$. This is given by the sum of the probability that $C_{1}$ survives till time $t$ and the probability that $C_{2}$ survives till time $t$, but we have to subtract from it the probability that both $C_{1}$ and $C_{2}$ survive till time $t$ since this will be counted twice otherwise. This can be seen from the Venn diagram of two objects shown in figure 10. Therefore, we have

$$
\widetilde{P}_{12}(t)=P_{1}(t)+P_{2}(t)-P_{12}(t, t) .
$$

From this we can compute the probability that the last one to survive decays between $t$ 
and $t+\delta t$ as

$$
-\delta t \frac{d}{d t} \widetilde{P}_{12}(t)
$$

Therefore, the life expectancy of the combined system is given by

$$
\bar{t}_{12}=-\int_{0}^{\infty} d t t \frac{d}{d t} \widetilde{P}_{12}(t, t)=\int_{0}^{\infty} d t\left\{P_{1}(t)+P_{2}(t)-P_{12}(t, t)\right\},
$$

where in the second step we have integrated by parts and used (3.26). Each of the first two integrals gives the result $\bar{t}_{1}$ computed in (3.17). For the last integral since we have to evaluate $P_{12}\left(t_{1}, t_{2}\right)$ at $t_{1}=t_{2}=t$ only the middle expression in (3.25) is relevant, and we get

$$
\begin{aligned}
\int_{0}^{\infty} P_{12}(t, t) d t & =\int_{0}^{\infty}(r+2)^{2 K} e^{-4 K t}\left(r+2 e^{-t}\right)^{-2 K} d t \\
& =2^{-4 K} r^{2 K}(r+2)^{2 K} B\left(\frac{2}{2+r} ; 4 K,-2 K\right) .
\end{aligned}
$$

Combining this with the result for $\bar{t}_{1}$ given in (3.17) and replacing $K$ by $1 / 2 T$ we get

$$
\begin{aligned}
\bar{t}_{12}= & 2(r+2)^{1 / T}\left[B\left(\frac{1}{2+r} ; \frac{1}{T}, 0\right)-B\left(\frac{1-r}{2} ; \frac{1}{T}, 0\right)+T \frac{(1-r)^{1 / T}}{2^{1 / T}}\right] \\
& -2^{-2 / T} r^{1 / T}(r+2)^{1 / T} B\left(\frac{2}{2+r} ; \frac{2}{T},-\frac{1}{T}\right) .
\end{aligned}
$$

We can now check various limits. First of all we can study the $r \rightarrow 0$ limit using the result

$$
B(x ; 2 \alpha,-\alpha) \simeq \frac{1}{\alpha}(1-x)^{-\alpha},
$$

for $x$ close to 1 . This gives $\lim _{r \rightarrow 0} \bar{t}_{12}=T$. This is in agreement with the fact that if the two objects remain at the same point then their combined life expectancy is the same as that of individual objects.

If on the other hand we take the limit of large $T$ then, using the result

$$
B(x ; \alpha, \beta) \simeq \frac{1}{\alpha}
$$

for small $\alpha$, we get $\bar{t}_{12} \simeq 3 T / 2$. Therefore, the life expectancy of the two objects together is $3 / 2$ times that of an isolated object. This is consistent with the fact that if the inverse decay rate of individual objects is large then typically there will be enough time for the two objects to go out of each other's horizon before they decay. Therefore, we can treat them as independent objects and recover the result (2.3). Mathematically this can be seen from the fact that when $T$ is large and $t \sim T$ then $P_{12}(t, t)$ given in the middle expression of (3.25) approaches $e^{-4 K t}$, which in turn is approximately equal to the square of $P_{1}(t)$ given in (3.14). 


\section{Vacuum decay in $3+1$ dimensional de Sitter space}

In this section we shall repeat the analysis of section 3 for $3+1$ dimensional de Sitter spacetime. Since the logical steps remain identical, we shall point out the essential differences arising in the two cases and then describe the results.

The metric of the $3+1$ dimensional de Sitter space is given by

$$
d s^{2}=-d t^{2}+e^{2 t}\left(d x^{2}+d y^{2}+d z^{2}\right)=\tau^{-2}\left(-d \tau^{2}+d x^{2}+d y^{2}+d z^{2}\right), \quad \tau \equiv-e^{-t} .
$$

There are of course various other coordinate systems in which we can describe the de Sitter metric, but the coordinate system used in (4.1) is specially suited for describing our universe, with $(x, y, z)$ labelling comoving coordinates and $t$ denoting the cosmic time in which the constant $t$ slices have uniform microwave background temperature. This form of the metric uses the observed flatness of the universe. The actual metric at present is deformed due to the presence of matter density, and also there is a lower cut-off on $t$ since our universe has a finite age of the order of the inverse Hubble constant. But both these effects will become irrelevant within a few Hubble time and we ignore them. In section 5.3 we shall study these effects, but at present our goal is to get an analytic result under these simplifying assumptions.

\subsection{Isolated comoving object}

First consider the case of an isolated object. The calculation proceeds as in section 3.1. However, in computing the volume of the past light cone in figure 3 we have to take into account the fact that for each $\sigma$, the light cone is a sphere of radius $(\tau-\sigma)$. Since the coordinate radius of the sphere is $(\tau-\sigma)$ and the space-time volume element scales as $1 / \sigma^{4}$ we get the volume of the past light cone of the object between $\tau$ and $\tau+\delta \tau$ to be

$$
\delta \tau \int_{-\infty}^{\tau} \frac{d \sigma}{\sigma^{4}} 4 \pi(\tau-\sigma)^{2}=-\frac{4}{3} \pi \tau^{-1} \delta \tau .
$$

This replaces the right hand side of (3.4). Therefore, (3.5) takes the form

$$
P_{0}^{\prime}(\tau)=\frac{4}{3} \pi \tau^{-1} K P_{0}(\tau)
$$

with the solution

$$
\begin{aligned}
\ln P_{0}(\tau) & =\frac{4}{3} \pi K \ln (-\tau), \\
P_{0}(t) & =\exp \left(-\frac{4}{3} \pi K t\right) .
\end{aligned}
$$

From this we can calculate the life expectancy of the isolated object to be

$$
T=\int_{0}^{\infty} P_{0}(t) d t=\frac{3}{4 \pi K} .
$$




\subsection{A pair of comoving objects}

The additional complication in the case of two objects comes from having to evaluate the contribution of the past light come of the first object between $\tau_{1}$ and $\tau_{1}+\delta \tau_{1}$ in situations depicted in figures 5 and 8 . Let us consider figure 8 since figure 5 can be considered as a special case of figure 8 with $\tau_{2}=-1$. Now in figure 8 which occurs for $\tau_{2}-r<\tau_{1}<\tau_{2}+r$, the past light cone of $C_{1}$ between $\tau_{1}$ and $\tau_{1}+\delta \tau_{1}$ lies partly inside the past light cone of $C_{2}$. We need to subtract this contribution from the total volume of the past light cone of $C_{1}$ between $\tau_{1}$ and $\tau_{1}+\delta \tau_{1}$, since the assumption that $C_{2}$ survives till $\tau_{2}$ rules out the formation of a bubble inside the past light cone of $C_{2}$. Our goal will be to calculate this volume.

Examining figure 8 we see that the intersection of the past light cones of $C_{1}$ at $\tau_{1}$ and $C_{2}$ at $\tau_{2}$ occur at $\tau=\sigma$ for $\sigma<\left(\tau_{1}+\tau_{2}-r\right) / 2$. At a value of $\sigma$ satisfying this constraint, the past light cone of $C_{1}$ at $\tau_{1}$ is a sphere of coordinate radius $r_{1}=\left(\tau_{1}-\sigma\right)$ and the past light cone of $C_{2}$ at $\tau_{2}$ is a sphere of coordinate radius $r_{2}=\left(\tau_{2}-\sigma\right)$. The centers of these spheres, lying at the comoving coordinates of the two objects have a coordinate separation of $r$. A simple geometric analysis shows that the coordinate area of the part of the first sphere that is inside the second sphere is given by

$$
\pi \frac{r_{1}}{r}\left\{r_{2}^{2}-\left(r_{1}-r\right)^{2}\right\}=\pi \frac{\left(\tau_{1}-\sigma\right)}{r}\left(\tau_{2}-\tau_{1}+r\right)\left(\tau_{1}+\tau_{2}-r-2 \sigma\right) .
$$

Taking into account the fact that physical volumes are given by $1 / \sigma^{4}$ times the coordinate volume we get the following expression for the volume of the past light cone of $C_{1}$ between $\tau_{1}$ and $\tau_{1}+\delta \tau_{1}$ that is inside the past light cone of $C_{2}$ :

$$
\begin{aligned}
\frac{\pi}{r}\left(\tau_{2}-\tau_{1}+r\right) \delta \tau_{1} \int_{-\infty}^{\left(\tau_{1}+\tau_{2}-r\right) / 2} & \frac{d \sigma}{\sigma^{4}}\left(\tau_{1}-\sigma\right)\left(\tau_{1}+\tau_{2}-r-2 \sigma\right) \\
= & \frac{2 \pi}{3 r}\left(\tau_{2}-\tau_{1}+r\right) \delta \tau_{1} \frac{\left(3 r-\tau_{1}-3 \tau_{2}\right)}{\left(r-\tau_{1}-\tau_{2}\right)^{2}}
\end{aligned}
$$

As already mentioned the excluded volume in case of figure 5 can be found by setting $\tau_{1}=\tau$ and $\tau_{2}=-1$ in (4.8).

We are now ready to generalize all the results of section 3. Let us begin with (3.12). Its generalization to the $3+1$ dimensional case takes the form

$$
\frac{d}{d \tau} \ln P_{1}(\tau)= \begin{cases}\frac{2 \pi K}{3}\left[\frac{2}{\tau}+\frac{(-1+r-\tau)(3+3 r-\tau)}{r(\tau-r-1)^{2}}\right] & \text { if } \tau<r-1 \\ \frac{4 \pi K}{3 \tau} & \text { if } \tau>r-1\end{cases}
$$


Its solution is given by

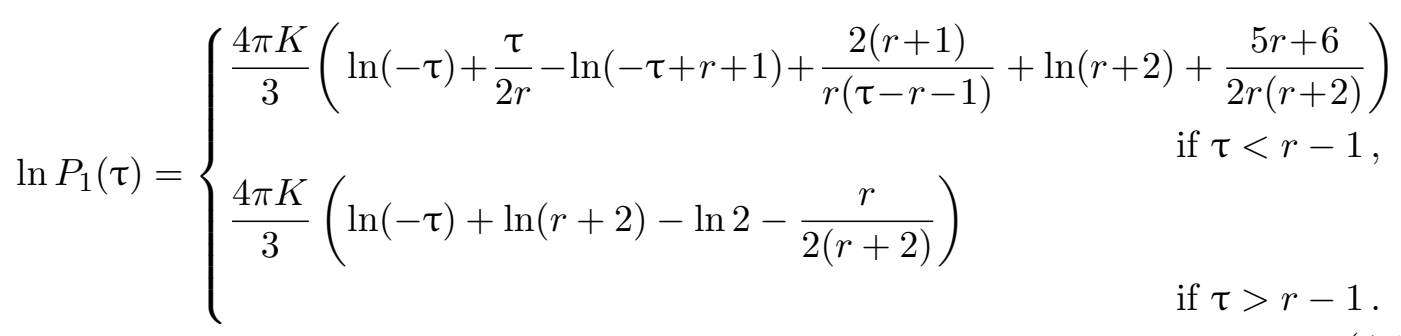

Expressing this in terms of $t$ using $\tau=-e^{-t}$ and $T \equiv 3 /(4 \pi K)$ we get

$$
P_{1}(t)=\left\{\begin{array}{rr}
\left(e^{-t}+r+1\right)^{-\frac{1}{T}}(r+2)^{\frac{1}{T}} \exp \left[-\frac{t}{T}+\frac{1}{T}\left\{-\frac{e^{-t}}{2 r}-\frac{2(r+1)}{r\left(e^{-t}+r+1\right)}+\frac{5 r+6}{2 r(r+2)}\right\}\right] \\
\text { for } t<-\ln (1-r), \\
\left(\frac{r+2}{2}\right)^{\frac{1}{T}} \exp \left[-\frac{t}{T}-\frac{r}{2 T(r+2)}\right] & \text { for } t>-\ln (1-r) .
\end{array}\right.
$$

The same expression holds for the survival probability $P_{2}(t)$ of $C_{2}$. From this we can find the life expectancy of $C_{1}$

$$
\bar{t}_{1}=\int_{0}^{\infty} P_{1}(t) d t .
$$

As in the $1+1$ dimensional case, $\bar{t}_{1}$ is slightly larger than $T$ but this is simply due to the choice of initial condition that both observers are assumed to exist at $t=0$. In figure 11 we have plotted the ratio $\bar{t}_{1} / T$ as a function of $T$ for various values of $r$, and as we can see the result remains close to 1 . More discussion on $\bar{t}_{1}$ can be found below (4.23).

Next we consider the generalization of (3.18)-(3.22). The analysis is straightforward and we get the results

$$
\begin{array}{lrl}
\frac{\partial \ln P_{12}\left(\tau_{1}, \tau_{2}\right)}{\partial \tau_{1}}=0 & \text { for } \quad \tau_{1}<\tau_{2}-r, \\
\frac{\partial \ln P_{12}\left(\tau_{1}, \tau_{2}\right)}{\partial \tau_{1}}=\frac{2 \pi K}{3}\left[\frac{2}{\tau_{1}}+\frac{\left(r-\tau_{1}+\tau_{2}\right)\left(3 r-\tau_{1}-3 \tau_{2}\right)}{r\left(r-\tau_{1}-\tau_{2}\right)^{2}}\right] & \text { for } \quad \tau_{2}-r<\tau_{1}<\tau_{2}+r \\
\frac{\partial \ln P_{12}\left(\tau_{1}, \tau_{2}\right)}{\partial \tau_{1}}=\frac{4 \pi K}{3 \tau_{1}} & \text { for } \quad \tau_{2}+r<\tau_{1}<0 .
\end{array}
$$

The solution to these equations, subject to the boundary condition $P_{12}\left(\tau_{1}=-1, \tau_{2}\right)=$ $P_{2}\left(\tau_{2}\right)=P_{1}\left(\tau_{2}\right)$ is given by

$$
\ln P_{12}\left(\tau_{1}, \tau_{2}\right)=\left\{\begin{array}{cl}
\frac{4 \pi K}{3}\left[\ln \left(-\tau_{2}\right)+\ln (r+2)-\frac{r}{2(r+2)}-\ln 2\right] \quad & \text { if } \tau_{1}<\tau_{2}-r \\
\frac{4 \pi K}{3}\left[\ln \left(-\tau_{1}\right)+\ln \left(-\tau_{2}\right)-\ln \left(-\tau_{1}-\tau_{2}+r\right)+\right. & \frac{\tau_{1}+\tau_{2}}{2 r} \\
\left.\quad-\frac{2 \tau_{1} \tau_{2}}{r\left(\tau_{1}+\tau_{2}-r\right)}+\ln (r+2)+\frac{1}{r+2}\right] \quad \text { if } \tau_{2}-r<\tau_{1}<\tau_{2}+r \\
\frac{4 \pi K}{3}\left[\ln \left(-\tau_{1}\right)+\ln (r+2)-\frac{r}{2(r+2)}-\ln 2\right] \quad \text { if } \tau_{2}+r<\tau_{1}<0
\end{array}\right.
$$




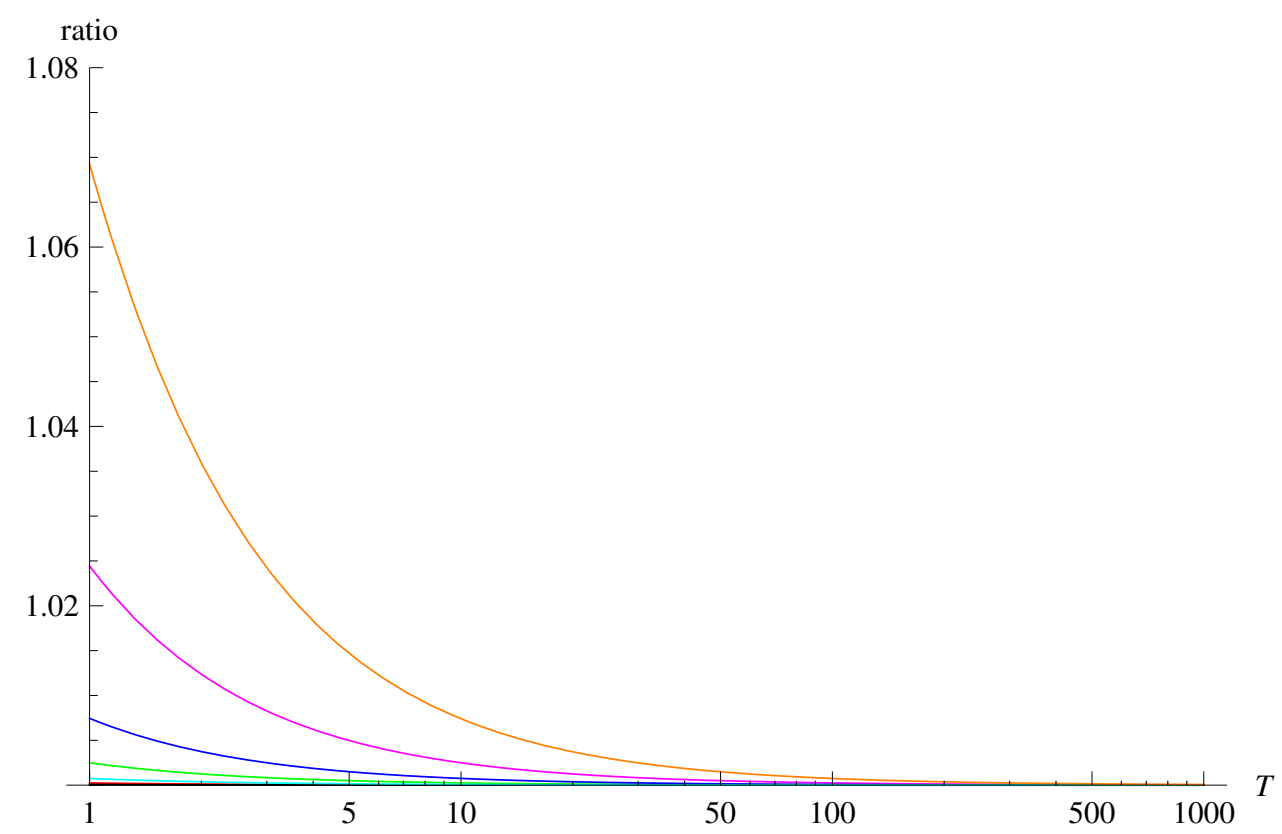

Figure 11. The figure showing the ratio $\bar{t}_{1} / T$ for $r=.0003, .001, .003, .01, .03, .1$ and .3 . For $r \leq .003$ the ratio is not distinguishable from 1 in this scale.

In terms of the physical time, and $T=3 /(4 \pi K)$, this becomes

$$
P_{12}\left(t_{1}, t_{2}\right)=\left\{\begin{array}{c}
\{(r+2) / 2\}^{1 / T} \exp \left[-\frac{r}{2 T(r+2)}-\frac{t_{2}}{T}\right] \\
\text { if } t_{1}<-\ln \left(r+e^{-t_{2}}\right) \\
(r+2)^{1 / T}\left(e^{-t_{1}}+e^{-t_{2}}+r\right)^{-\frac{1}{T}} \exp \left[\frac{1}{T(r+2)}-\frac{1}{T}\left(t_{1}+t_{2}\right)\right. \\
\left.-\frac{1}{2 T r}\left(e^{-t_{1}}+e^{-t_{2}}\right)+\frac{2}{T r} \frac{1}{e^{t_{1}}+e^{t_{2}}+r e^{t_{1}+t_{2}}}\right] \\
\text { if }-\ln \left(r+e^{-t_{2}}\right)<t_{1}<-\ln \left(e^{-t_{2}}-r\right) \\
\{(r+2) / 2\}^{1 / T} \exp \left[-\frac{r \quad}{2 T(r+2)}-\frac{t_{1}}{T}\right] \\
\text { if } t_{1}>-\ln \left(e^{-t_{2}}-r\right)
\end{array}\right.
$$

This gives

$$
P_{12}(t, t)=(r+2)^{1 / T} e^{\frac{1}{T(r+2)}}\left(2 e^{-t}+r\right)^{-\frac{1}{T}} \exp \left[-\frac{2}{T} t-\frac{1}{T r} e^{-t}+\frac{2}{T r} \frac{1}{2 e^{t}+r e^{2 t}}\right] .
$$

In terms of this, and the functions $P_{1}=P_{2}$ given in (4.11), we can calculate the probability $\widetilde{P}_{12}$ of at least one of the two objects surviving till time $t$ using

$$
\widetilde{P}_{12}(t)=P_{1}(t)+P_{2}(t)-P_{12}(t, t)
$$


and the combined life expectancy of two objects using the analog of (3.28)

$$
\bar{t}_{12}=\int_{0}^{\infty} \widetilde{P}_{12}(t) d t=\int_{0}^{\infty} d t\left\{P_{1}(t)+P_{2}(t)-P_{12}(t, t)\right\}=2 \bar{t}_{1}-\int_{0}^{\infty} d t P_{12}(t, t) .
$$

For the integral of $P_{12}(t, t)$ one can write down an expression in terms of special functions as follows. Defining $y$ via

$$
2+r e^{t}=\frac{2+r}{y}
$$

for $r \neq 0$, we get

$$
\begin{aligned}
\int_{0}^{\infty} d t P_{12}(t, t)=[r(r+ & \left.2)^{-1}\right]^{1 / T} e^{\frac{1}{(r+2) T}} \\
& \times \int_{0}^{1} d y y^{-1+2 / T}\left(1-\frac{2 y}{2+r}\right)^{-1-1 / T} \exp \left[-\frac{y}{(2+r) T}\right] .
\end{aligned}
$$

Now, using the result

$$
\int_{0}^{1} d y \frac{y^{a-1}(1-y)^{c-a-1}}{(1-u y)^{b}} e^{v y}=B(a, c-a) \Phi_{1}(a, b, c ; u, v)
$$

with $\operatorname{Re} c>\operatorname{Re} a>0,|u|<1, B$ the beta function and $\Phi_{1}$ the confluent hypergeometric series of two variables (Humbert series), we get

$$
\int_{0}^{\infty} d t P_{12}(t, t)=\frac{T}{2}\left[\frac{r}{(r+2)}\right]^{1 / T} e^{\frac{1}{T(r+2)}} \Phi_{1}\left(\frac{2}{T}, 1+\frac{1}{T}, 1+\frac{2}{T} ; \frac{2}{2+r},-\frac{1}{(2+r) T}\right) .
$$

$\Phi_{1}$ has a power series expansion

$$
\Phi_{1}(a, b, c ; u, v)=\sum_{m, n=0}^{\infty} \frac{(a)_{m+n}(b)_{m}}{(c)_{m+n} m ! n !} u^{m} v^{n}, \quad|u|<1
$$

where $(a)_{m} \equiv a(a+1) \cdots(a+m-1)$.

Unfortunately we have not been able to find an expression for $\bar{t}_{1}=\int_{0}^{\infty} d t P_{1}(t)$ in terms of special functions. However, we can write down a series expansion for this that will be suitable for studying its behaviour for small $r$. The integral of (4.11) from $t=-\ln (1-r)$ to $\infty$ is straightforward and yields

$$
T(1-r)^{1 / T}(r+2)^{1 / T} 2^{-1 / T} \exp \left[-\frac{r}{2 T(r+2)}\right] .
$$

The integral of (4.11) from $t=0$ to $-\ln (1-r)$ can be analyzed by making a change of variable from $t$ to $y$ via $e^{-t}=(1-y r)$. In terms of this variable the integral can be expressed as

$$
\begin{aligned}
& r \int_{0}^{1} d y\left(1-\frac{y r}{r+2}\right)^{-1 / T}(1-y r)^{-1+1 / T} \times \\
& \times \exp \left[-\frac{2 y^{2} r(r+1)}{T(2+r)^{3}}\left(1-\frac{y r}{2+r}\right)^{-1}\right] \exp \left[\frac{y r^{2}}{2 T(2+r)^{2}}\right] .
\end{aligned}
$$


Using series expansion of the second and third terms in the integrand we get

$$
\begin{aligned}
\sum_{m, n=0}^{\infty} \frac{1}{m ! n !}(1 & \left.-\frac{1}{T}\right)_{m}(-1)^{n} \frac{2^{n} r^{m+n+1}(r+1)^{n}}{T^{n}(2+r)^{3 n}} \\
& \times \int_{0}^{1} d y y^{m+2 n}\left(1-\frac{y r}{2+r}\right)^{-n-1 / T} \exp \left[\frac{y r^{2}}{2 T(2+r)^{2}}\right]
\end{aligned}
$$

The integral over $y$ can be expressed in terms of $\Phi_{1}$ using (4.21). Adding (4.24) to this we get

$$
\begin{aligned}
\bar{t}_{1}=T(1-r)^{1 / T}(r+2)^{1 / T} 2^{-1 / T} \exp \left[-\frac{r}{2 T(r+2)}\right] \\
+\sum_{m, n=0}^{\infty} \frac{1}{m ! n !} \frac{1}{m+2 n+1}\left(1-\frac{1}{T}\right)_{m}(-1)^{n} \frac{2^{n} r^{m+n+1}(r+1)^{n}}{T^{n}(2+r)^{3 n}} \\
\quad \times \Phi_{1}\left(m+2 n+1, n+\frac{1}{T}, m+2 n+2 ; \frac{r}{2+r}, \frac{r^{2}}{2 T(2+r)^{2}}\right) .
\end{aligned}
$$

It can be checked using (4.11), (4.12), (4.16) and (4.18) that for $r \rightarrow 0$ we get $\bar{t}_{12} / \bar{t}_{1}=1$ and for $T \rightarrow \infty$ we get $\bar{t}_{12} / \bar{t}_{1}=3 / 2$. The values of 'gain' $\equiv \bar{t}_{12} / \bar{t}_{1}$ for different values of $r$ have been plotted against $T$ in figure 1.

\subsection{The case of small initial separation}

Since from practical considerations the small $r$ region is of interest, it is also useful to consider the expansion of $\bar{t}_{12} / \bar{t}_{1}$ for small $r$. For this we have to analyze the behaviour of $\bar{t}_{1}$ as well as that of $\int_{0}^{\infty} d t P_{12}(t, t)$ for small $r$. Let us begin with $\bar{t}_{1}$ given in (4.27). It can be easily seen that this is given by $T+\mathcal{O}(r)$ with the contribution $T$ coming from the first term. However, the contribution from $\int_{0}^{\infty} d t P_{12}(t, t)$ has a more complicated behaviour at small $r$. This is related to the fact that in the $r \rightarrow 0$ limit the fourth argument of $\Phi_{1}$ in (4.22) approaches 1 , and in this limit the series expansion (4.23) diverges. To study the small $r$ behaviour we shall go back to the original expression for $P_{12}(t, t)$ given in (4.16). We change variable to $v=e^{-t} / r$ and write

$$
\begin{aligned}
\int_{0}^{\infty} d t P_{12}(t, t)= & (r+2)^{1 / T} e^{1 / T(r+2)} r^{1 / T} \int_{0}^{1 / r} \frac{d v}{v}(2 v+1)^{-1 / T} v^{2 / T} \exp \left[-\frac{v}{T(2 v+1)}\right] \\
= & (r+2)^{1 / T} e^{1 / T(r+2)} r^{1 / T} \int_{0}^{1 / r} \frac{d v}{v} v^{2 / T}\left[(2 v+1)^{-1 / T} \exp \left[-\frac{v}{T(2 v+1)}\right]\right. \\
& \left.-(2 v)^{-1 / T} \exp \left[-\frac{1}{2 T}\right]\right]+(r+2)^{1 / T} 2^{-1 / T} \exp \left[\frac{1}{T(r+2)}-\frac{1}{2 T}\right] T
\end{aligned}
$$

where in the last step we have subtracted an integral from the original integral and compensated for it by adding the explicit result for the integral. This subtraction makes the integral convergent even when we replace the upper limit $1 / r$ by $\infty$. Taking the small $r$ 


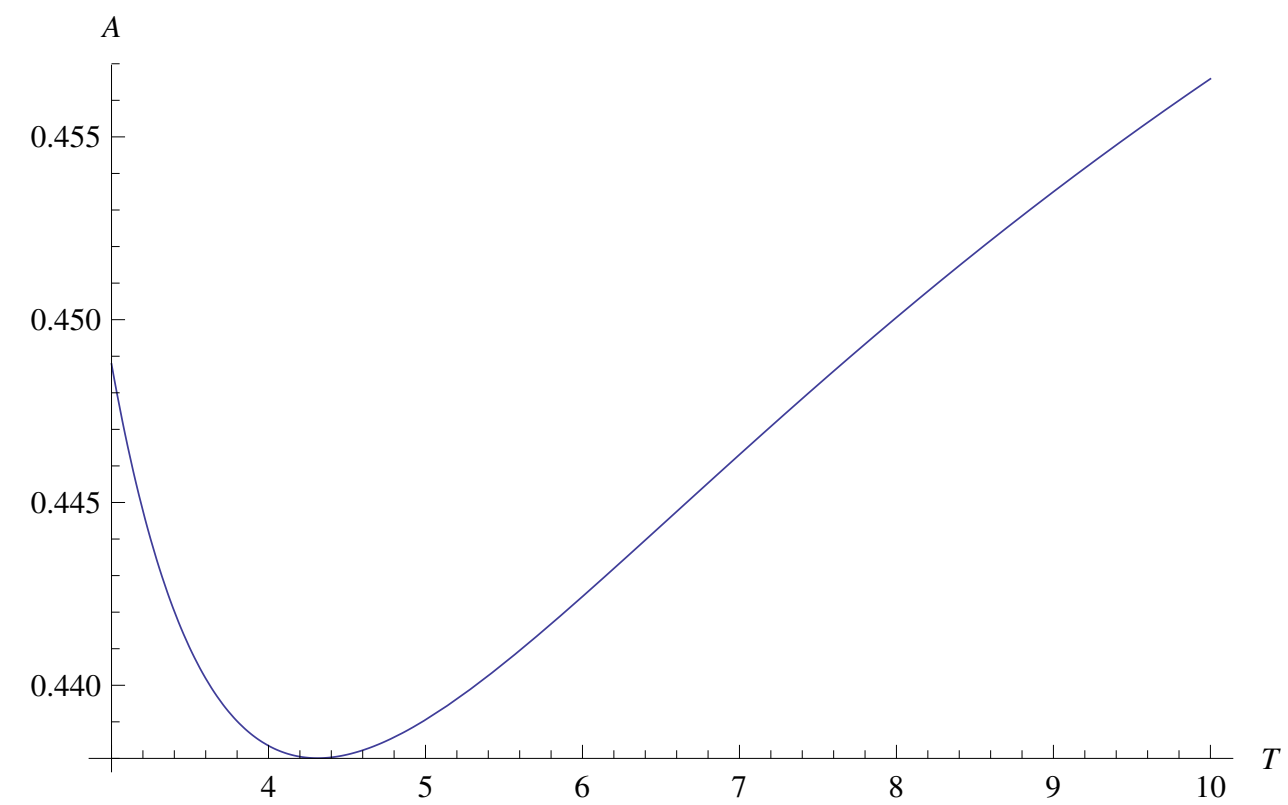

Figure 12. The coefficient of the $r^{1 / T}$ term in the expression for $\bar{t}_{12} / \bar{t}_{1}$ as a function of $T$.

limit we get

$$
\begin{aligned}
\int_{0}^{\infty} d t P_{12}(t, t)= & 2^{1 / T} r^{1 / T} \int_{0}^{\infty} \frac{d v}{v} v^{2 / T}\left[(2 v+1)^{-1 / T} \exp \left[-\frac{v}{T(2 v+1)}+\frac{1}{2 T}\right]-(2 v)^{-1 / T}\right] \\
& +T+\mathcal{O}(r) .
\end{aligned}
$$

Combining this with the earlier result that for $r \rightarrow 0, \bar{t}_{1} \simeq T+\mathcal{O}(r)$ and using (4.18) we get

$$
\frac{\bar{t}_{12}}{\bar{t}_{1}}=2-\frac{1}{\bar{t}_{1}} \int_{0}^{\infty} d t P_{12}(t, t)=1+A(T) r^{1 / T}
$$

where

$$
A(T)=T^{-1} 2^{1 / T} \int_{0}^{\infty} d v v^{-1+2 / T}\left[(2 v)^{-1 / T}-(2 v+1)^{-1 / T} \exp \left(-\frac{v}{T(2 v+1)}+\frac{1}{2 T}\right)\right] .
$$

The numerical values of $A(T)$ are moderate - for example $A(5) \simeq 0.439$ and $A(10) \simeq 0.457$. A plot of $A(T)$ as a function of $T$ has been shown in figure 12. The $1 / T$ exponent of $r$ shows that even if we begin with small $r$, for moderately large $T$ (say $T \sim 5$ ) we can get moderate enhancement in life expectancy.

\section{Generalizations}

In this section we shall discuss various possible generalizations of our results.

\subsection{Multiple objects in de Sitter space}

We shall begin by discussing the case of three objects $C_{1}, C_{2}$ and $C_{3}$ placed at certain points in $3+1$ dimensional de Sitter space-time and analyze the probability that at least 


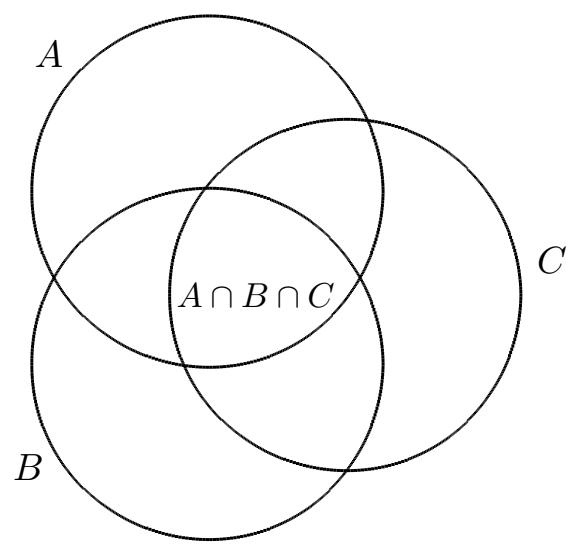

Figure 13. The Venn diagram illustrating that the survival probability of one of $A, B$ or $C$, denoted by $P(A \cup B \cup C)$, is given by $P(A)+P(B)+P(C)-P(A \cap B)-P(B \cap C)-P(A \cap C)+P(A \cap B \cap C)$.

one of them will survive till time $t$. Let $P_{123}\left(t_{1}, t_{2}, t_{3}\right)$ denote the probability that $C_{1}$ survives till time $t_{1}, C_{2}$ survives till time $t_{2}$ and $C_{3}$ survives till time $t_{3}$. Similarly $P_{i j}\left(t_{i}, t_{j}\right)$ for $1 \leq i, j \leq 3$ will denote the probability that $C_{i}$ survives till $t_{i}$ and $C_{j}$ survives till $t_{j}$ and $P_{i}\left(t_{i}\right)$ will denote the probability that $C_{i}$ survives till $t_{i}$. All probabilities are defined under the prior assumption that all objects are alive at $t=0$. These probabilities can be calculated by generalizing the procedure described in section 3 and section 4 by constructing ordinary differential equations in one of the arguments at fixed values of the other arguments. The geometry of course now becomes more involved due to the fact that the past light cone of one object will typically intersect the past light cones of the other objects which themselves may have overlaps, and one has to carefully subtract the correct volume. But the analysis is straightforward.

The quantity of direct interest is the probability $\widetilde{P}_{123}(t)$ that at least one of the objects survives till time $t$. With the help of the Venn diagram given in figure 13 we get

$$
\widetilde{P}_{123}(t)=\left(P_{1}(t)+P_{2}(t)+P_{3}(t)-P_{12}(t, t)-P_{13}(t, t)-P_{23}(t, t)+P_{123}(t, t, t)\right) .
$$

Using this we can calculate the life expectancy of the combined system as

$$
-\int_{0}^{\infty} d t t \frac{d}{d t} \widetilde{P}_{123}(t)=\int_{0}^{\infty} d t \widetilde{P}_{123}(t) .
$$

The generalization to the case of $N$ objects is now obvious. The relevant formula is

$$
\widetilde{P}_{12 \cdots N}(t)=\left(\sum_{i=1}^{N} P_{i}(t)-\sum_{i<j}^{N} P_{i j}(t, t)+\sum_{i<j<k}^{N} P_{i j k}(t, t, t)+\cdots(-1)^{N+1} P_{12 \cdots N}(t, t, \cdots, t)\right)
$$

where $P_{i_{1} \cdots i_{k}}\left(t_{i_{1}}, \cdots t_{i_{k}}\right)$ are again computed by solving ordinary differential equations in one of the variables. Once $\widetilde{P}_{12 \cdots N}(t)$ is computed we can get the life expectancy of the combined system by using

$$
\bar{t}_{12 \cdots N}=\int_{0}^{\infty} \widetilde{P}_{12 \cdots N}(t) d t
$$




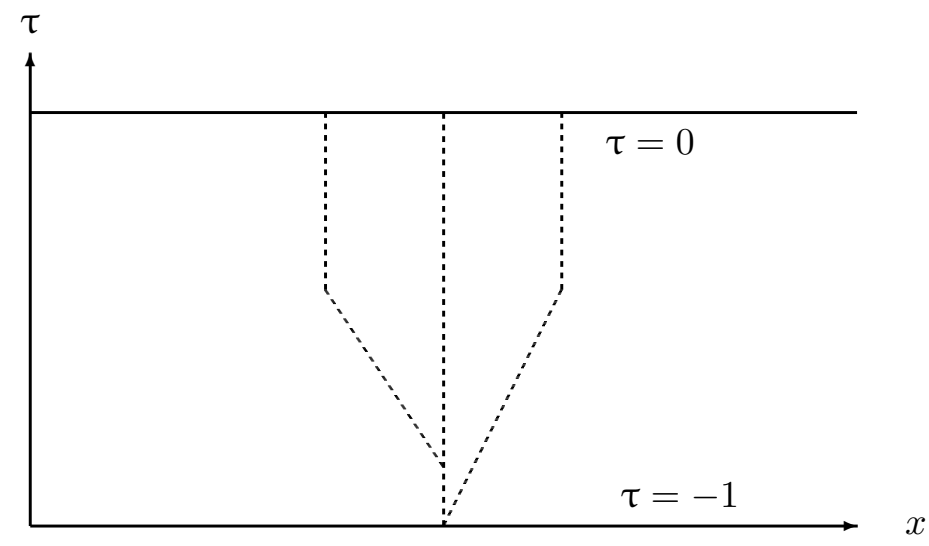

Figure 14. Multiple objects originating from the same space-time point. Different dashed lines represent the trajectories followed by different objects.

\subsection{Realistic trajectories}

Another generalization involves considering a situation where multiple objects originate at the same space time point and then follow different trajectories, eventually settling down at different comoving coordinates. This has been illustrated in figure 14. This represents the realistic situation since by definition different civilizations of the same race must originate at some common source. We can now generalize our analysis to take into account the possibility of decay during the journey as well. Eqs. (5.3) and (5.4) still holds, but the computation of $P_{i_{1} \cdots i_{k}}\left(t_{i_{1}}, \cdots t_{i_{k}}\right)$ will now have to be done by taking into account the details of the trajectories of each object and the overlaps of their past light cones. The principle remains the same, and we can set up ordinary differential equations for each of these quantities. The only difference is that the spatial separation between the $i$-th object at $\tau=\tau_{i}$ and the $j$-th object at $\tau=\tau_{j}$ will now depend on $\tau_{i}$ and $\tau_{j}$ according to the trajectories followed by them.

This analysis can be easily generalized to the case where each of the descendant objects in turn produces its own descendants which settle away from the parent object and eventually go outside each other's horizon due to the Hubble expansion. If this could be repeated at a rate faster than the vacuum decay rate then we can formally ensure that some of the objects will survive vacuum decay [16]. However, since within a few Hubble periods most of the universe will split up into gravitationally bound systems outside each other's horizon, in practice this is going to be an increasingly difficult task.

\subsection{Matter effect}

A third generalization will involve relaxing the assumption that the universe has been de Sitter throughout its past history. While de Sitter metric will be a good approximation after a few Hubble periods, within the next few Hubble periods we shall still be sensitive to the fact that the universe had been matter dominated in the recent past and had a 
beginning. This will change the form of the metric (4.1) to

$$
d s^{2}=-d t^{2}+a(t)^{2}\left(d x^{2}+d y^{2}+d z^{2}\right)
$$

where $a(t)$ is determined from the Friedman equation

$$
\frac{1}{a} \frac{d a}{d t}=\sqrt{\frac{8 \pi G}{3}\left(\rho_{\Lambda}+\frac{\rho_{m}}{a^{3}}\right)}
$$

in the convention that the value of $a$ is 1 today and $\rho_{\Lambda}$ and $\rho_{m}$ are the energy densities due to cosmological constant and matter today. Since we have chosen the unit of time so that the Hubble parameter in the cosmological constant dominated universe is 1, we have $\sqrt{8 \pi G \rho_{\Lambda} / 3}=1$. Defining ${ }^{6}$

$$
c \equiv \rho_{m} / \rho_{\Lambda} \simeq 0.45
$$

we can express (5.6) as

$$
\frac{1}{a} \frac{d a}{d t}=\sqrt{1+c a^{-3}} .
$$

Let $\tau$ be the conformal time defined via

$$
d \tau=d t / a(t)
$$

with the boundary condition $\tau \rightarrow 0$ as $t \rightarrow \infty$. Then (5.6) takes the form

$$
\frac{1}{a^{2}} \frac{d a}{d \tau}=\sqrt{1+c a^{-3}}
$$

whose solution is

$$
\tau=-\int_{a}^{\infty} \frac{d b}{b^{2} \sqrt{1+c b^{-3}}}=-\int_{0}^{1 / a} \frac{d v}{\sqrt{1+c v^{3}}}=-\frac{1}{a}{ }_{2} F_{1}\left(\frac{1}{3}, \frac{1}{2} ; \frac{4}{3},-\frac{c}{a^{3}}\right) .
$$

This implicitly determines $a$ as a function of $\tau$. The metric is given by

$$
d s^{2}=a(\tau)^{2}\left(-d \tau^{2}+d x^{2}+d y^{2}+d z^{2}\right) .
$$

Using the experimental value $c \simeq 0.45$ we get that $\tau \rightarrow \tau_{0} \simeq-3.7$ as $a \rightarrow 0$, showing that the big bang singularity is at $\tau \simeq-3.7$. $^{7}$ We also have that at $a=1, \tau \simeq-0.95$. This is not very different from the value $\tau=-1$ for pure de Sitter space-time with which we have worked. However, we shall now show that the decay rate in the matter dominated epoch of the universe differs significantly from that in the cosmological constant dominated epoch. The decay rate of an isolated observer at some value of the conformal time $\tau$ is given by the following generalization of (4.2), (4.3):

$$
\begin{aligned}
\frac{d}{d \tau} \ln P_{0}(\tau) & =-4 \pi K \int_{\tau_{0}}^{\tau} d \sigma a(\sigma)^{4}(\tau-\sigma)^{2} \\
& =-4 \pi K \int_{b=0}^{a(\tau)} \frac{d b}{b^{2} \sqrt{1+c b^{-3}}} b^{4}\left\{\tau+\frac{1}{b}{ }_{2} F_{1}\left(\frac{1}{3}, \frac{1}{2} ; \frac{4}{3},-\frac{c}{b^{3}}\right)\right\}^{2}
\end{aligned}
$$

\footnotetext{
${ }^{6}$ We use cosmological parameters given in [18].

${ }^{7}$ Of course close to the singularity the universe becomes radiation dominated but given the short span of radiation dominated era we ignore that effect for the current analysis.
} 
where in the second step we have changed the integration variable from $\sigma$ to $b=a(\sigma)$. From this we can compute the decay rate:

$$
\begin{aligned}
D(t) & \equiv-\frac{d}{d t} \ln P_{0}=-\frac{1}{a(t)} \frac{d}{d \tau} \ln P_{0}(\tau) \\
& =\frac{4 \pi K}{a(t)} \int_{b=0}^{a(t)} \frac{d b}{\sqrt{1+c b^{-3}}} b^{2}\left\{\tau(t)+\frac{1}{b}{ }_{2} F_{1}\left(\frac{1}{3}, \frac{1}{2} ; \frac{4}{3},-\frac{c}{b^{3}}\right)\right\}^{2} .
\end{aligned}
$$

Using the information that today $a=1$ and $\tau \simeq-0.95$ we get

$$
\left.D(t)\right|_{\text {today }} \simeq \frac{4 \pi K}{3} \times 0.067 \simeq \frac{0.067}{T} .
$$

This is lower than the corresponding rate $T^{-1}$ in the de Sitter epoch by about a factor of 15. The growth of the decay rate with scale factor has been shown in figure 2 .

Our analysis of section 4 can now be repeated for two or more observers and also for general trajectory discussed in section 5.2 with this general form of the metric to get more accurate computation of the life expectancy. These corrections will be important if $T \lesssim 1$ and the decay takes place within a few Hubble period from now. On the other hand if $T$ is large (say $\gtrsim 10$ ) then the decay is likely to take place sufficiently far in the future by which time the effect of our matter dominated past will have insignificant effect on the results.

The fact that the decay rate increases with time till it eventually settles down to a constant value in the de Sitter epoch has some important consequences:

1. We have already seen from (5.15) that the decay rate today is about 15 times smaller than the decay rate in the de Sitter epoch. Eq. (5.14) for $a(t)=2$ shows that even when the universe will be double its size compared to today, the decay rate will remain at about $27 \%$ of the decay rate in the de Sitter epoch. Since most of the journeys to different parts of the universe - if they take place at all — are likely to happen during this epoch, we see that the probability of decay during the journey will be considerably less than that in the final de Sitter phase. This partially justifies our analysis in section 4 where we neglected the probability of decay during the journey. This also shows that if we eventually carry out a detailed numerical analysis taking into account the effect discussed in section 5.2, it should be done in conjunction with the analysis of this subsection taking into account the effect of matter.

2. It is also possible to see from (5.14), (5.15) (or figure 2) that the decay rate in the past was even smaller than that of today. If $D(t)$ denotes the decay rate at time $t$ defined in (5.14), then the average decay rate in our past can be defined as

$$
\frac{1}{t_{1}} \int_{0}^{t_{1}} D(t) d t
$$

where $t_{1}$ denotes the current age of the universe given by

$$
t_{1}=\int_{0}^{1} d a\left(\frac{d a}{d t}\right)^{-1}=\int_{0}^{1} \frac{d a}{a \sqrt{1+c a^{-3}}} \simeq 0.79 .
$$


In physical units $t_{1}$ is about $1.38 \times 10^{10}$ years. The evaluation of (5.16) can be facilitated using the observation that $D(t) d t$ is $K$ times the volume enclosed between the past light cones of the object at times $t$ and $t+d t$. Therefore, $\int_{0}^{t_{1}} d t D(t)$ must be $K$ times the total volume enclosed by the past light cone of the object at $t_{1}$. This can be easily computed, yielding

$$
\begin{aligned}
\int_{0}^{t_{1}} D(t) d t & =\frac{4}{3} \pi K \int_{\tau_{0}}^{\tau} d \sigma a(\sigma)^{4}(\tau-\sigma)^{3} \\
& =\frac{1}{T} \int_{b=0}^{a(\tau)} \frac{d b}{b^{2} \sqrt{1+c b^{-3}}} b^{4}\left\{\tau+\frac{1}{b}{ }_{2} F_{1}\left(\frac{1}{3}, \frac{1}{2} ; \frac{4}{3},-\frac{c}{b^{3}}\right)\right\}^{3} .
\end{aligned}
$$

For $c \simeq 0.45$ and $\tau$ given by today's value -0.95 this gives

$$
\int_{0}^{t_{1}} D(t) d t=\frac{0.014}{T}
$$

Using (5.16), (5.17) we get the average decay rate to be $0.018 / T$. This is about 3.7 times smaller than the present decay rate given in (5.15) and 56 times smaller than the decay rate $1 / T$ in the de Sitter epoch.

Integrating the equation $d P_{0} / d t=-D(t) P_{0}(t)$ we get

$$
\ln P_{0}\left(t_{1}\right)=-\int_{0}^{t_{1}} d t D(t)=-\frac{0.014}{T} .
$$

Requiring this to be not much smaller than -1 (which is equivalent to requiring that the inverse of the average decay rate (5.16) be not much smaller than the age of the universe $t_{1}$ ) gives $T \gtrsim 0.014$. This is much lower than what one might have naively predicted by equating the lower bound on $T$ to the age of the universe i.e. $T \gtrsim t_{1} \sim 0.79$. Recalling that the unit of time is set by the Hubble period in the de Sitter epoch which is about $1.7 \times 10^{10}$ years, the bound $T \gtrsim 0.014$ translates to a lower bound of order $2.5 \times 10^{8}$ years. Since the current decay rate is about 15 times smaller than that in the final de Sitter epoch, we see that the lower bound on the current inverse decay rate is of order $3.7 \times 10^{9}$ years. This is comparable to the period over which the earth is expected to be destroyed due to the expanded size of the Sun.

3. Finally we note that the above analysis was based on the assumption that the bubbles continue to nucleate and expand in the FRW metric at the same rate as they would do in the metastable vacuum. This will be expected as long as the matter and radiation density and temperature are small compared to the microscopic scales involved in the bubble nucleation process, e.g. the scale set by the negative cosmological constant of the vacuum in the interior of the bubble. Some discussion on the effect of cosmological space-time background on the bubble nucleation / evolution can be found in [19, 20].

\section{Discussion}

We have seen that the result for how much we can increase the life expectancy by spreading out in space depends on the parameters $r$ and $K$, which in turn are determined by the 
Hubble parameter of the de Sitter space-time, the initial spread between different objects and the inverse decay rate. Therefore, the knowledge of these quantities is important for planning our future course of action if we are to adapt this strategy for increasing the life expectancy of the human race. In this section we shall discuss possible strategies for determining / manipulating these quantities.

We begin with the Hubble expansion parameter $H$. This is determined by the cosmological constant which has been quite well measured by now. Assuming that the current expansion rate is of order $68 \mathrm{Km} / \mathrm{sec} / \mathrm{Mpc}$ and accounting for the fact that the cosmological constant accounts for about $69 \%$ of the total energy density we get $H^{-1} \simeq 1.7 \times 10^{10}$ years. Future experiments will undoubtedly provide a more accurate determination of this number, but given the uncertainty in the other quantities, this will not significantly affect our future course of action. Of course we may discover that the dark energy responsible for the accelerated expansion of the universe comes from another source, in which case we have to reexamine the whole situation.

Next we turn to the initial separation between different objects which determine the value of $r$. Since in order for the Hubble expansion to be effective in separating the objects they have to be unbound gravitationally, a minimum separation between the objects is necessary for overcoming the attractive gravitational force of the home galaxy. For example the size of our local gravitationally bound group of galaxies is of order 5 million light years which correspond to $r \sim 3 \times 10^{-4}$. The question is whether larger values of $r$ can be accessed. An interesting analysis by Heyl [21] concluded that by building a space-ship that can constantly accelerate / decelerate at a value equal to the acceleration due to gravity, we can reach values of $r$ close to unity in less than 100 years viewed from the point of view of the space-traveller. Of course this will be close to about $10^{10}$ years viewed from earth, and roughly the reduction of time viewed from the space-ship can be attributed to the large time dilation at the peak speed of the space-ship reaching a value close to that of light. However, this large time dilation will also increase the effective temperature of the microwave background radiation in the forward direction and without a proper shield such a journey will be impossible to perform. If one allows a maximum time dilation of the order of 100 then the microwave temperature in the forward direction rises to about the room temperature. Even then we have to worry about the result of possible collisions with intergalactic dust and other debris in space. Even if these problems are resolved, we shall need a time of order $10^{8}$ years from the point of view of the space-ship to travel a distance of order $10^{10}$ light years. Even travelling the minimum required distance of order $10^{7}$ light years will take $10^{5}$ years in such a space-ship. Such a long journey in a space-ship does not seem very practical but may not be impossible.

Another interesting suggestion for populating regions of space-time which will eventually be outside each other's horizon has been made by Loeb [22]. Occasionally there are hypervelocity stars which escape our galaxy (and the cluster of galaxies which are gravitationally bound) and so if we could find a habitable planet in such a star we could take a free ride in that planet and escape our local gravitationally bound system. In general of course there is no guarantee that such a star will reach another cluster of galaxies where we could spread out and thrive, but some time we may be lucky. It has been further suggested 
in $[23,24]$ that the merger of Andromeda and the Milky Way galaxies in the future [25] could generate a large number of such hypervelocity stars travelling at speeds comparable to that of light and they could travel up to distances of the order of $10^{9}$ light years by the time they burn out. This could allow us to achieve values of $r$ of order $10^{-1}$ or more.

Let us now turn to the value of $T$ or equivalently the decay rate of the de Sitter vacuum in which we currently live. This is probably the most important ingredient since we have seen that for $T \lesssim 1$ we do not gain much by spreading out, while for large enough $T$ we can achieve the maximum possible gain, given by the harmonic numbers, by spreading out even over modest distances of $r \sim 10^{-3}$. At the same time if $T$ is so large that it exceeds the period over which galaxies will die then vacuum decay may not have a significant role in deciding our end and we should focus on other issues. For this reason estimating the value of $T$ seems to be of paramount importance. Unfortunately, due to the very nature of the vacuum decay process it is not possible to determine it by any sort of direct experiment since such an experiment will also destroy the observer. It may be possible in the future to device clever indirect experiments to probe vacuum instability without actually causing the transition to the stable vacuum, but no such scheme is known at present.

At a crude level the current age of the universe - which is about 0.79 times the asymptotic Hubble period in the cosmological constant dominated epoch - together with the assumption that we have not been extremely lucky to survive this long, suggests that the inverse decay rate of the universe is $\gtrsim 0$.8. However, (5.18) shows that this actually gives a lower bound of $T \gtrsim 0.014$ reflecting the fact that the decay rate in the de Sitter epoch will be about 56 times faster than the average decay rate in the past. If we allow for the possibility that we might have been extremely lucky to have survived till today, then we have indirect arguments that lower the bound by a factor of 10 [13]. Clearly these rates are too fast and if $T$ really happens to be less than 1, then there is not much we can do to prolong our collective life.

Is there any hope of computing $T$ theoretically? Unfortunately any bottom up approach based on the analysis of low energy effective field theory is insufficient for this problem. The reason for this is that the vacuum decay rate is a heavily ultraviolet sensitive quantity. Given a theory with a perfectly stable vacuum we can add to it a new heavy scalar field whose effect will be strongly suppressed at low energy, but which can have a potential that makes the vacuum metastable with arbitrarily large decay rate. For this reason the only way we could hope to estimate $T$ is through the use of a top down approach in which we have a fundamental microscopic theory all of whose parameters are fixed by some fundamental principle, and then compute the vacuum decay rate using standard techniques. In the context of string theory this will require finding the vacuum in the landscape that describes our universe. Alternatively, in the multiverse scenario, we need to carry out a statistical analysis that establishes that the overwhelming majority of the vacua that resemble our vacua will have their decay rate lying within a narrow range. This can then be identified as the likely value of the decay rate. There have been attempts in this direction [26-29], but it is probably fair to say that we do not yet have a definite result based on which we can plan our future course of action. 


\section{Acknowledgments}

We would like to thank Adam Brown, Rajesh Gopakumar and Abraham Loeb for useful discussions. This work was supported in part by the DAE project 12-R\&D-HRI-5.020303. The work of A.S. was also supported in part by the J. C. Bose fellowship of the Department of Science and Technology, India and the KIAS distinguished professorship. The work of MV was also supported by the SPM research grant of the Council for Scientific and Industrial Research (CSIR), India.

\section{A Decay rate for equation of state $p=w \rho$}

In this appendix we shall compute the growth of decay rate with time for a general equation of state of the form $p=w \rho$ with $w>-1$. In this case the $\rho$ and $a$ are related as

$$
\rho=\rho_{0} a^{-3(w+1)},
$$

for some constant $\rho_{0}$. As a result the dependence of $a$ on $t$ and $\tau$ are determined by the equations

$$
\frac{1}{a} \frac{d a}{d t}=\sqrt{\frac{8 \pi G}{3} \rho}=C a^{-3(w+1) / 2}, \quad C \equiv \sqrt{\frac{8 \pi G}{3} \rho_{0}},
$$

and

$$
\frac{1}{a^{2}} \frac{d a}{d \tau}=C a^{-3(w+1) / 2} .
$$

The solutions to these equations are

$$
t=\frac{2}{3 C(w+1)} a^{3(w+1) / 2}, \quad \tau=\frac{2}{(3 w+1) C} a^{(3 w+1) / 2} .
$$

As $a$ ranges from 0 to $\infty$, both $t$ and $\tau$ also range from 0 to $\infty$.

We can now compute the decay rate using the first equation of (5.13):

$$
\begin{aligned}
D(t) & \equiv-\frac{1}{a} \frac{d}{d \tau} \ln P_{0}(\tau) \\
& =\frac{1}{a} 4 \pi K \int_{0}^{\tau} d \sigma a(\sigma)^{4}(\tau-\sigma)^{2},
\end{aligned}
$$

where $a(\sigma)$ denotes the scale factor at conformal time $\sigma$. Changing integration variable to $b=a(\sigma)$, which due to (A.4) corresponds to

$$
\sigma=\frac{2}{(3 w+1) C} b^{(3 w+1) / 2},
$$

we can express (A.5) as

$$
\begin{aligned}
D(t) & =4 \pi K a^{-1}\left(\frac{2}{(3 w+1) C}\right)^{3} \frac{3 w+1}{2} \int_{0}^{a} d b b^{4} b^{(3 w-1) / 2}\left(a^{(3 w+1) / 2)}-b^{(3 w+1) / 2}\right)^{2} \\
& =\frac{32 \pi K}{3 C^{3}} a^{9(w+1) / 2} \frac{1}{(w+3)(3 w+5)(9 w+11)} .
\end{aligned}
$$

Using the relation between $a$ and $t$ given in (A.4), this can be expressed as

$$
D(t)=36 \pi K \frac{(w+1)^{3}}{(w+3)(3 w+5)(9 w+11)} t^{3} .
$$


Open Access. This article is distributed under the terms of the Creative Commons Attribution License (CC-BY 4.0), which permits any use, distribution and reproduction in any medium, provided the original author(s) and source are credited.

\section{References}

[1] I.Yu. Kobzarev, L.B. Okun and M.B. Voloshin, Bubbles in metastable vacuum, Sov. J. Nucl. Phys. 20 (1975) 644 [INSPIRE].

[2] M. Stone, The lifetime and decay of excited vacuum states of a field theory associated with nonabsolute minima of its effective potential, Phys. Rev. D 14 (1976) 3568 [INSPIRE].

[3] P.H. Frampton, Consequences of vacuum instability in quantum field theory, Phys. Rev. D 15 (1977) 2922 [INSPIRE].

[4] S.R. Coleman, The fate of the false vacuum. 1. Semiclassical theory, Phys. Rev. D 15 (1977) 2929 [Erratum ibid. D 16 (1977) 1248] [INSPIRE].

[5] C.G. Callan Jr. and S.R. Coleman, The fate of the false vacuum. 2. First quantum corrections, Phys. Rev. D 16 (1977) 1762 [INSPIRE].

[6] S.R. Coleman and F. De Luccia, Gravitational effects on and of vacuum decay, Phys. Rev. D 21 (1980) 3305 [INSPIRE].

[7] Supernova Search Team collaboration, A.G. Riess et al., Observational evidence from supernovae for an accelerating universe and a cosmological constant, Astron. J. 116 (1998) 1009 [astro-ph/9805201] [INSPIRE].

[8] Supernova Cosmology Project collaboration, S. Perlmutter et al., Measurements of $\Omega$ and $\Lambda$ from 42 high redshift supernovae, Astrophys. J. 517 (1999) 565 [astro-ph/9812133] [INSPIRE].

[9] R. Bousso and J. Polchinski, Quantization of four form fluxes and dynamical neutralization of the cosmological constant, JHEP 06 (2000) 006 [hep-th/0004134] [INSPIRE].

[10] S.B. Giddings, S. Kachru and J. Polchinski, Hierarchies from fluxes in string compactifications, Phys. Rev. D 66 (2002) 106006 [hep-th/0105097] [INSPIRE].

[11] S. Kachru, R. Kallosh, A.D. Linde and S.P. Trivedi, De Sitter vacua in string theory, Phys. Rev. D 68 (2003) 046005 [hep-th/0301240] [INSPIRE].

[12] N. Bostrom, Anthropic bias: observation selection effects in science and philosophy, Routledge, New York U.S.A. (2002).

[13] M. Tegmark and N. Bostrom, How unlikely is a doomsday catastrophe?, astro-ph/0512204 [INSPIRE].

[14] A. Masoumi, Topics in vacuum decay, arXiv:1505.06397.

[15] A.H. Guth, The inflationary universe: a possible solution to the horizon and flatness problems, Phys. Rev. D 23 (1981) 347 [INSPIRE].

[16] A. Sen, Riding gravity away from doomsday, arXiv:1503.08130 [INSPIRE].

[17] L.M. Krauss and G.D. Starkman, Life, the universe and nothing: life and death in an ever expanding universe, Astrophys. J. 531 (2000) 22 [astro-ph/9902189] [INSPIRE].

[18] Planck collaboration, P.A.R. Ade et al., Planck 2015 results. XIII. Cosmological parameters, arXiv:1502.01589 [INSPIRE]. 
[19] W. Fischler, S. Paban, M. Zanic and C. Krishnan, Vacuum bubble in an inhomogeneous cosmology: a toy model, JHEP 05 (2008) 041 [arXiv: 0711.3417] [INSPIRE].

[20] D. Simon, J. Adamek, A. Rakic and J.C. Niemeyer, Tunneling and propagation of vacuum bubbles on dynamical backgrounds, JCAP 11 (2009) 008 [arXiv:0908.2757] [INSPIRE].

[21] J.S. Heyl, The long-term future of space travel, Phys. Rev. D 72 (2005) 107302 [astro-ph/0509268] [INSPIRE].

[22] A. Loeb, Cosmology with hypervelocity stars, JCAP 04 (2011) 023 [arXiv:1102.0007] [INSPIRE].

[23] J. Guillochon and A. Loeb, The fastest unbound stars in the universe, Astrophys. J. 806 (2015) 124 [arXiv:1411.5022] [INSPIRE].

[24] A. Loeb and J. Guillochon, Observational cosmology with semi-relativistic stars, arXiv: 1411.5030 [INSPIRE].

[25] K. Nagamine and A. Loeb, Future evolution of nearby large scale structure in a universe dominated by a cosmological constant, New Astron. 8 (2003) 439 [astro-ph/0204249] [INSPIRE].

[26] T. Clifton, S. Shenker and N. Sivanandam, Volume weighted measures of eternal inflation in the Bousso-Polchinski landscape, JHEP 09 (2007) 034 [arXiv:0706.3201] [INSPIRE].

[27] M. Dine, G. Festuccia, A. Morisse and K. van den Broek, Metastable domains of the landscape, JHEP 06 (2008) 014 [arXiv:0712.1397] [INSPIRE].

[28] D.N. Page, Possible anthropic support for a decaying universe: a cosmic doomsday argument, arXiv:0907.4153 [INSPIRE].

[29] A.R. Brown and A. Dahlen, Giant leaps and minimal branes in multi-dimensional flux landscapes, Phys. Rev. D 84 (2011) 023513 [arXiv:1010.5241] [InSPIRE]. 